\title{
COMPARING DUSHNIK-MILLER DIMENSION, BOOLEAN DIMENSION AND LOCAL DIMENSION
}

\author{
FIDEL BARRERA-CRUZ*, THOMAS PRAG, HEATHER C. SMITH* ${ }^{*}$ LIBBY TAYLOR*, \\ AND WILLIAM T. TROTTER
}

\begin{abstract}
The original notion of dimension for posets is due to Dushnik and Miller and has been studied extensively in the literature. Quite recently, there has been considerable interest in two variations of dimension known as Boolean dimension and local dimension. For a poset $P$, the Boolean dimension of $P$ and the local dimension of $P$ are both bounded from above by the dimension of $P$ and can be considerably less. Our primary goal will be to study analogies and contrasts among these three parameters. As one example, it is known that the dimension of a poset is bounded as a function of its height and the tree-width of its cover graph. The Boolean dimension of a poset is bounded in terms of the tree-width of its cover graph, independent of its height. We show that the local dimension of a poset cannot be bounded in terms of the tree-width of its cover graph, independent of height. We also prove that the local dimension of a poset is bounded in terms of the path-width of its cover graph. In several of our results, Ramsey theoretic methods will be applied.
\end{abstract}

\section{INTRODUCTION}

We investigate combinatorial problems for finite posets. As has become standard in the literature, we use the terms elements and points interchangeably in referring to the members of the ground set of a poset. We write $x \| y$ in $P$ when $x$ and $y$ are incomparable in a poset $P$, and we let $\operatorname{Inc}(P)$ denote the set of all ordered pairs $(x, y)$ with $x \| y$ in $P$. As a binary relation, $\operatorname{Inc}(P)$ is symmetric. Recall that a non-empty family $\mathcal{R}$ of linear extensions of $P$ is called a realizer of $P$ when $x<y$ in $P$ if and only if $x<y$ in $L$ for each $L \in \mathcal{R}$. Clearly, a non-empty family $\mathcal{R}$ of linear extensions of $P$ is a realizer of $P$ if and only if for each $(x, y) \in \operatorname{Inc}(P)$, there is some $L \in \mathcal{R}$ for which $x>y$ in $L$. The dimension of a poset $P$, denoted $\operatorname{dim}(P)$, as defined by Dushnik and Miller in their seminal paper [6], is the least positive integer $d$ for which $P$ has a realizer $\mathcal{R}$ with $|\mathcal{R}|=d$.

For an integer $n \geq 2$, the standard example $S_{n}$ is the height 2 poset with minimal elements $A=\left\{a_{1}, a_{2}, \ldots, a_{n}\right\}$ and maximal elements $B=\left\{b_{1}, b_{2}, \ldots, b_{n}\right\}$. Furthermore, $a_{i}<b_{j}$ in $S_{n}$ if and only if $i \neq j$. As noted in [6], $\operatorname{dim}\left(S_{n}\right)=n$, for all

Date: June 19, 2019.

2010 Mathematics Subject Classification. 06A07, 05C35, 05D10.

Key words and phrases. dimension, Boolean dimension, local dimension, tree-width, path-width, Ramsey theory.

*Much of the research was completed while affiliated with the Georgia Instutite of Technology. 
$n \geq 2$. Also, dimension is clearly a monotonic parameter, i.e., if $Q$ is a subposet of $P$, then $\operatorname{dim}(Q) \leq \operatorname{dim}(P)$. Accordingly, a poset which contains a large standard example as a subposet has large dimension. On the other hand, there are posets which do not contain the standard example $S_{2}$ as a subposet and nevertheless have large dimension. This observation is the poset analogue to the fact that there are triangle-free graphs which have large chromatic number.

Quite recently, researchers have been investigating combinatorial problems for two variations of the Dushnik-Miller concept for dimension, known as Boolean dimension and local dimension. The concept of Boolean dimension was introduced by Gambosi, Nešetřil and Talamo in a 1987 conference paper [9], with the full version [10] appearing in journal form in 1990. However, we use here the definition of Boolean dimension which appears in a 1989 paper by Nešetřil and Pudlák [28]. This paper was first presented in conference form in 1987. Later, we will comment on the distinction between the two definitions.

On the other hand, the quite new notion of local dimension is due to Torsten Ueckerdt [37] who shared his ideas with participants of the workshop on Order and Geometry held in Gułtowy, Poland, September 14-17, 2016. Ueckerdt's new concept resonated with researchers at the workshop, and it served to kindle renewed interest in Boolean dimension as well.

Here is the definition for Boolean dimension. For a positive integer $d$, let $\mathbf{2}^{d}$ denote the set of all $0-1$ strings of length $d$. Such strings are also called bit strings. Let $P$ be a poset and let $\mathcal{B}=\left\{L_{1}, L_{2}, \ldots, L_{d}\right\}$ be a non-empty family of linear orders on the ground set of $P$ (these linear orders need not be linear extensions of $P$ ). Also, let $\tau: \mathbf{2}^{d} \rightarrow\{0,1\}$ be a function. For each pair $(x, y)$ of distinct elements of $P$, we form a bit string $q(x, y, \mathcal{B})$ of length $d$ which has value 1 in coordinate $i$ if and only if $x<y$ in $L_{i}$. The pair $(\mathcal{B}, \tau)$ is a Boolean realizen 1 when for each pair $x, y$ of distinct elements of $P, x<y$ in $P$ if and only if $\tau(q(x, y, \mathcal{B}))=1$. The Boolean dimension of $P$, denoted $\operatorname{bdim}(P)$, is the least positive integer $d$ for which $P$ has a Boolean realizer $(\mathcal{B}, \tau)$ with $|\mathcal{B}|=d$. Clearly, $\operatorname{bdim}(P) \leq \operatorname{dim}(P)$, since if $\mathcal{R}=\left\{L_{1}, L_{2}, \ldots, L_{d}\right\}$ is a realizer of $P$, we simply take $\tau$ as the function which maps $(1,1, \ldots, 1)$ to 1 while all other bit strings of length $d$ are mapped to 0 .

Trivially, $\operatorname{bdim}(P)=1$ if and only if $P$ is either a chain or an antichain 2 . Also, $\operatorname{bdim}(Q) \leq \operatorname{bdim}(P)$ when $Q$ is a subposet of $P$. Clearly, $\operatorname{bdim}(P)=\operatorname{bdim}\left(P^{*}\right)$ where $P^{*}$ denotes the dual of $P$. It is an easy exercise to show that if $\operatorname{bdim}(P)=2$, then $\operatorname{dim}(P)=2$. In [10], Gambosi, Nešetřil and Talamo show that $\operatorname{dim}(P)=3$ if and only if $\operatorname{bdim}(P)=3$. However, their proof uses a more restrictive definition of Boolean dimension. In [35, Trotter and Walczak simplify the proof given in [10] and

\footnotetext{
${ }^{1}$ In [10], a pair $(\mathcal{B}, \tau)$ with $\mathcal{B}=\left\{L_{1}, L_{2}, \ldots, L_{d}\right\}$, is considered a Boolean realizer only when there is some $i$ for which $L_{i}$ is a linear extension of $P$ and $\tau(x, y)=1$ implies $x<y$ in $L_{i}$. We prefer to drop both these restrictions, as is done in [28].

${ }^{2}$ In [7, Felsner, Mészáros and Micek consider pairs $x, y$ of not necessarily distinct elements of $P$ so a query $q(x, y, \mathcal{B})$ has coordinate $i$ set to 1 if and only if $x \leq y$ in $L_{i}$. With this restriction, the function $\tau$ is constrained to send the constant string $(1,1, \ldots, 1)$ to 1 , so that a non-trivial antichain has Boolean dimension 2. For all other posets, their definition and ours give exactly the same value for Boolean dimension.
} 
show that it actually works for the more general notion of Boolean dimension we are studying in this paper. It is an easy exercise to show that all standard examples have Boolean dimension at most 4 . In fact, $\operatorname{bdim}\left(S_{n}\right)=n$ when $2 \leq n \leq 4$ and $\operatorname{bdim}\left(S_{n}\right)=4$ when $n \geq 4$.

Here is the definition for local dimension. Let $P$ be a poset. A partial linear extension, abbreviated ple, of $P$ is a linear extension of a subposet of $P$. Whenever $\mathcal{L}$ is a family of ple's of $P$ and $u \in P$, we set $\mu(u, \mathcal{L})=|\{L \in \mathcal{L}: u \in L\}|$. In turn, we set $\mu(\mathcal{L})=\max \{\mu(u, \mathcal{L}): u \in P\}$. A non-empty family $\mathcal{L}$ of ple's of a poset $P$ is called a local realizer of $P$ if the following two conditions are satisfied:

(1) If $x \leq y$ in $P$, there is some $L \in \mathcal{L}$ for which $x \leq y$ in $L$;

(2) if $(x, y) \in \operatorname{Inc}(P)$, there is some $L \in \mathcal{L}$ for which $x>y$ in $L$.

The local dimension of $P$, denoted $\operatorname{ldim}(P)$, is defined as

$$
\lim (P)=\min \{\mu(\mathcal{L}): \mathcal{L} \text { is a local realizer of } P\} .
$$

Clearly, $\lim (P) \leq \operatorname{dim}(P)$ for all posets $P$, since any realizer is also a local realizer. Also, $\operatorname{ldim}(P)=1$ if and only if $P$ is a chain; $\operatorname{ldim}(Q) \leq \operatorname{ldim}(P)$ if $Q$ is a subposet of $P$; and if $P^{*}$ is the dual of $P$, then $\operatorname{ldim}\left(P^{*}\right)=\operatorname{ldim}(P)$. It is an easy exercise to show that if $\operatorname{ldim}(P)=2$, then $\operatorname{dim}(P)=2$. In presenting his concept to conference participants, Ueckerdt [37] noted that the local dimension of a standard example is at most 3. In fact, $\operatorname{ldim}\left(S_{n}\right)=n$ when $2 \leq n \leq 3$ and $\operatorname{ldim}\left(S_{n}\right)=3$ when $n \geq 3$.

In this paper, we give analogies and contrasts between (Dushnik-Miller) dimension, Boolean dimension and local dimension. Although our results touch on several other topics, we consider the connections with structural graph theory, given in Section 5, our main theorems. A number of open problems remain, and we give a summary listing in the closing section.

Our arguments will use the following notational conventions:

(1) If $n$ is a positive integer, then we use the now standard notation $[n]$ to represent $\{1,2, \ldots, n\}$.

(2) Let $\mathcal{L}=\left\{L_{1}, L_{2}, \ldots, L_{t}\right\}$ be a family of ple's of a poset $P$. If $x \in P$, and $\mu(x, \mathcal{L})=m$, then there are integers $j_{1}<j_{2}<\cdots<j_{m}$ so that $x$ is in $L_{j_{\alpha}}$ for each $\alpha \in[m]$. In this case, we will say that occurrence $\alpha$ of $x$ is in $L_{j_{\alpha}}$.

(3) We will make use of the general form of Ramsey's theorem: For every triple $(k, h, r)$ of positive integers with $h \geq k$, there is a least positive integer $\operatorname{Ram}(k, h ; r)$ so that if $n \geq \operatorname{Ram}(k, h ; r)$ and $\varphi$ is any coloring of the $k$ element subsets of $[n]$ using $r$ colors, then there is an $h$-element subset $H$ of $[n]$ so that $\varphi$ maps all $k$-element subsets of $H$ to the same color.

\section{Forcing Large Boolean Dimension and Large local Dimension}

Since standard examples have small Boolean dimension and small local dimension, it is of interest to explore what can cause these two parameters to be large. We start with an example of a well known family of posets where dimension, local dimension and Boolean dimension all grow together.

When $n \geq 2$, we let $I_{n}$ denote the canonical interval order whose elements are the closed intervals of the form $[i, j]$ where $i$ and $j$ are integers with $1 \leq i<j \leq n$. The 
partial order on $I_{n}$ is defined by setting $[i, j]<[k, l]$ in $I_{n}$ when $j<k$. As is well known, the poset $I_{n}$ does not contain the standard example $S_{2}$, but the dimension of $I_{n}$ goes to infinity with $n$. In fact, the value of $\operatorname{dim}\left(I_{n}\right)$ is now known to within an additive constant (see the remarks in [2]). We now explain why both $\operatorname{ldim}\left(I_{n}\right)$ and $\operatorname{bdim}\left(I_{n}\right)$ tend to infinity. We start with the result for local dimension.

Theorem 2.1. For each $s \geq 1$, if $n \geq \operatorname{Ram}\left(4,7 ; s^{2}\right)$, then $\operatorname{ldim}\left(I_{n}\right)>s$.

Proof. Suppose to the contrary that for some $s \geq 1$, and $n \geq \operatorname{Ram}\left(4,7 ; s^{2}\right)$ we have $\operatorname{ldim}\left(I_{n}\right) \leq s$. Let $\mathcal{L}=\left\{L_{i}: 1 \leq i \leq t\right\}$ be a local realizer for $I_{n}$ with $\mu\left(I_{n}, \mathcal{L}\right) \leq s$. Consider a 4-element subset $\{a, b, c, d\}$ of $[n]$ with $a<b<c<d$. Then there is some least positive integer $m \in[t]$ so that $[a, c]>[b, d]$ in $L_{m}$. We then set $\varphi(\{a, b, c, d\})=(\alpha, \beta)$ where occurrence $\alpha$ of $[a, c]$ is in $L_{m}$ and occurrence $\beta$ of $[b, d]$ is in $L_{m}$. Now, we have a coloring of the 4-element subsets of $[n]$ using $s^{2}$ colors.

In view of our choice for the size of $n$, we know there is some 7-element subset $H=\{a, b, c, d, e, f, g\}$ of $[n]$ and a color $(\alpha, \beta)$ so that all 4-element subsets of $H$ are mapped to $(\alpha, \beta)$. We may assume, without loss of generality, that $a<b<$ $c<d<e<f<g$. Now consider the subset $\{a, c, d, g\}$. Then let $m$ be the least positive integer so that $[a, d]>[c, g]$ in $L_{m}$. Then occurrence $\alpha$ of $[a, d]$ is in $L_{m}$ as is occurrence $\beta$ of $[c, g]$.

Now consider the set $\{b, c, f, g\}$. Since occurrence $\beta$ of $[c, g]$ is in $L_{m}$, then the least $m^{\prime}$ such that $[b, f]>[c, g]$ in $L_{m^{\prime}}$ is $m^{\prime}=m$ and occurrence $\alpha$ of $[b, f]$ is also in $L_{m}$.

Now consider the set $\{b, e, f, g\}$. Since occurrence $\alpha$ of $[b, f]$ is in $L_{m}$, we know that occurrence $\beta$ of $[e, g]$ is also in $L_{m}$. Furthermore, we know that $[b, f]>[e, g]$ in $L_{m}$. Finally, we consider the set $\{a, b, d, f\}$ and conclude that $[a, d]>[b, f]$ in $L_{m}$. In particular $\alpha=\beta$. However, we have now shown that $[a, d]>[b, f]>[e, g]$ in $L_{m}$. This is a contradiction since $[a, d]<[e, g]$ in $I_{n}$.

Here is the analogous result for Boolean dimension.

Theorem 2.2. For each $d \geq 1$, if $n \geq \operatorname{Ram}\left(4,6 ; 2^{d}\right)$, then $\operatorname{bdim}\left(I_{n}\right)>d$.

Proof. Suppose to the contrary that for some $d \geq 1$, and $n \geq \operatorname{Ram}\left(4,6 ; 2^{d}\right)$ we have $\operatorname{bdim}\left(I_{n}\right) \leq d$. Let $(\mathcal{B}, \tau)$ be a Boolean realizer for $I_{n}$ with $\mathcal{B}=\left\{L_{1}, L_{2}, \ldots, L_{d}\right\}$. Then for each 4-element subset $\{a, b, c, d\}$ of $[n]$ with $a<b<c<d$, we define the coloring $\varphi$ by setting $\varphi(\{a, b, c, d\})=q([a, c],[b, d], \mathcal{B})$.

In view of our choice for $n$, we may assume that there is some binary string $\sigma$ of length $d$ and a 6 -element subset $H=\{a, b, c, d, e, f\}$ of $[n]$, such that $a<b<$ $c<d<e<f$, so that $\varphi$ maps all 4-element subsets of $H$ to $\sigma$. In particular, $\varphi$ assigns the color $\sigma$ to the 4-element subsets $\{a, b, c, e\}$ and $\{b, d, e, f\}$, that is, $q([a, c],[b, e], \mathcal{B})=\sigma=q([b, e],[d, f], \mathcal{B})$.

Now let $i \in[d]$. If $\sigma(i)=1$, then $[a, c]<[b, e]<[d, f]$ in $L_{i}$. If $\sigma(i)=0$, then $[d, f]<[b, e]<[a, c]$ in $L_{i}$. However, this shows that $\sigma=q([a, c],[b, e], \mathcal{B})=$ $q([a, c],[d, f], \mathcal{B})$. This is a contradiction since $[a, c]<[d, f]$ in $I_{n}$, so $\tau(q([a, c],[d, f], \mathcal{B}))=$ 1 , but $[a, c]$ and $[b, e]$ are incomparable, so $\tau(q([a, c],[b, e], \mathcal{B}))=0$. 
Next, we present a family for which dimension and local dimension are unbounded but Boolean dimension is bounded. For a pair $(d, n)$ of integers with $2 \leq d<n$, let $P(1, d ; n)$ denote the poset consisting of all 1-element and $d$-element subsets of $[n]$ partially ordered by inclusion. We abbreviate the dimension, Boolean dimension and local dimension of $P(1, d ; n)$ as $\operatorname{dim}(1, d ; n), \operatorname{bdim}(1, d ; n)$ and $\operatorname{ldim}(1, d ; n)$, respectively. Dushnik [5] calculated $\operatorname{dim}(1, d ; n)$ exactly when $d \geq 2 \sqrt{n}$, and Spencer [29] showed that for fixed $d, \operatorname{dim}(1, d ; n)=\Theta(\log \log n)$. Historically, there has been considerable interest in the case where $d=2$. Combining results of Hoşten and Morris [13] with estimates of Kleitman and Markovsky [23, the following theorem follows easily (see the comments in [2]).

Theorem 2.3. For every $\epsilon>0$, there is an integer $n_{0}$ so that if $n>n_{0}$ and

$$
s=\lg \lg n+1 / 2 \lg \lg \lg n+1 / 2 \lg \pi+1 / 2,
$$

then $s-\epsilon<\operatorname{dim}(1,2 ; n)<s+1+\epsilon$.

As a consequence, for almost all large values of $n$, we can compute the value of $\operatorname{dim}(1,2 ; n)$ exactly; for the remaining small fraction of values, we are able to compute two consecutive integers and say that $\operatorname{dim}(1,2 ; n)$ is one of the two.

We are not able to compute the value of $\operatorname{ldim}(1,2 ; n)$ as accurately, but at least we can show that $\operatorname{ldim}(1,2 ; n)$ goes to infinity with $n$.

Theorem 2.4. For each $s \geq 1$, if $n \geq \operatorname{Ram}\left(3,4 ; s^{2}\right)$, then $\operatorname{ldim}(1,2 ; n)>s$.

Proof. Fix $s \geq 1$ and let $n \geq \operatorname{Ram}\left(3,4 ; s^{2}\right)$. We assume that $\mathcal{L}=\left\{L_{1}, L_{2}, \ldots, L_{t}\right\}$ is a local realizer for $P=P(1,2 ; n)$ with $\mu(P, \mathcal{L}) \leq s$ and argue to a contradiction. In the argument, we abbreviate the singleton sets in $P(1,2 ; n)$ by omitting braces, i.e., the singleton set $\{a\}$ will just be written as $a$. Now the partial order is that an integer $a \in[n]$ is less than a 2 -element set $S$ in $P(1,2 ; n)$ when $a \in S$.

Now let $T=\{a, b, c\}$ be a 3 -element subset of $[n]$. We may assume without loss of generality that $a<b<c$. Since $b \notin\{a, c\}$, there is some least integer $m \in[t]$ with $b>\{a, c\}$ in $L_{m}$. Then there is an ordered pair $(\alpha, \beta) \in[s] \times[s]$ of (not necessarily distinct) integers so that occurrence $\alpha$ of $b$ is in $L_{m}$ and occurrence $\beta$ of $\{a, c\}$ is in $L_{m}$. We then have a coloring $\varphi$ of the 3 -element subsets of $[n]$ using $s^{2}$ colors. Since $n \geq \operatorname{Ram}\left(3,4 ; s^{2}\right)$, there is some color $(\alpha, \beta)$ and a 4-element subset $H=\{a, b, c, d\}$ so that all 3 -element subsets of $H$ are assigned color $(\alpha, \beta)$. Again, we may assume without loss of generality that $a<b<c<d$.

We consider first the 3 -element subset $\{a, b, d\}$ and note that there is some $m \in[t]$ for which $b>\{a, d\}$ in $L_{m}$. Furthermore, occurrence $\alpha$ of $b$ is in $L_{m}$ while occurrence $\beta$ of $\{a, d\}$ is in $L_{m}$. Now consider the subset $\{a, c, d\}$. Since occurrence $\beta$ of $\{a, d\}$ is in $L_{m}$, we must have occurrence $\alpha$ of $c$ in $L_{m}$ with $c>\{a, d\}$ in $L_{m}$.

Now consider the subset $\{a, b, c\}$. Since occurrence $\alpha$ of $b$ is in $L_{m}$, we must then have $b>\{a, c\}$ in $L_{m}$. On the other hand, if we consider the subset $\{b, c, d\}$, since occurrence $\alpha$ of $c$ is in $L_{m}$, we must have $c>\{b, d\}$ in $L_{m}$. We then have $\{b, d\}<c<\{a, c\}<b$ in $L_{m}$, which is a contradiction to the fact that $b<\{b, d\}$ in every ple of $P(1,2 ; n)$ where $b$ and $\{b, d\}$ appear. 
Since $P(1,2 ; n)$ is a subposet of $P(1, d ; n+d-2)$, it follows that for fixed $d \geq 2$, $\operatorname{ldim}(1, d ; n)$ tends to infinity with $n$. However, as we will soon see $\operatorname{bdim}(1, d ; n)$ is bounded in terms of $d$.

For the family $P(1, d ; n)$, every maximal element is comparable with exactly $d$ elements. A careful reading of the proof of Theorem 3.6 on page 259 in [10] shows that they have actually established the following result.

Theorem 2.5. Let $P$ be a poset of height 2. If there is some positive integer $d$ so that each maximal element of $P$ is comparable with at most $d$ minimal elements, then $\operatorname{bdim}(P) \leq 2 d$.

The inequality in Theorem 2.5 is obviously tight for $d=1$. We will now show that it is tight for $d \geq 2$. To accomplish, we will show that $\operatorname{bdim}(1, d ; n)=2 d$, provided $n$ is sufficiently large in terms of $d$. The argument will make use of the following elementary observation. When $(\mathcal{B}, \tau)$ is a Boolean realizer of a poset $P$, it is easy to see that a linear order $L_{i}$ in $\mathcal{B}$ can be replaced $\sqrt{3}$ with $L_{i}^{*}$, the dual of $L_{i}$, i.e., $x<y$ in $L_{i}$ if and only if $x>y$ in $L_{i}^{*}$. Of course, we must also make the obvious modification to the map $\tau$.

Theorem 2.6. For each $d \geq 2$, there is some positive integer $n_{0}$ so that if $n \geq n_{0}$, then $\operatorname{bdim}(1, d ; n)=2 d$.

Proof. We already know that $\operatorname{bdim}(1, d ; n) \leq 2 d$ for all $d \geq 2$. We fix a value of $d \geq 2$, suppose that $\operatorname{bdim}(1, d ; n)<2 d$ for each $n>d$ and argue to a contradiction.

Let $(\mathcal{B}, \tau)$ be a Boolean realizer for $P(1, d ; n)$ with $\mathcal{B}=\left\{L_{1}, L_{2}, \ldots, L_{s}\right\}$ such that $s<2 d$. As before, we take $\operatorname{Min}(P)=[n]$ with $\operatorname{Max}(P)$ the family of all $d$-element subsets of $[n]$.

First, we apply Erdôs-Szekeres to the set $[n]$ of minimal elements of $P$ relative to the order of these elements in the linear orders in $\mathcal{B}$ to obtain a subset $A$ of $[n]$ that appears either in increasing order or decreasing order for each $L_{i} \in \mathcal{B}$. Using our previous remarks concerning duals of linear orders in $\mathcal{B}$, if $n$ is sufficiently large, we may assume there is a subset $A$ of $[n]$ with $|A|=2 d+1$ so that the restriction of $L_{j}$ to $A$ is exactly the same as the restriction of $L_{k}$ to $A$ whenever $1 \leq j<k \leq s$. After relabeling, we may assume $A=\{1,2, \ldots, 2 d+1\}$ so that $1<2<3<\cdots<2 d+1$ in $L_{j}$ for each $j=1,2, \ldots, s$.

There are $2 d$ "gaps" between consecutive elements of $A$ of the form $(i, i+1)$. One of $i$ and $i+1$ is even and the other is odd. Now consider the maximal element $S=\{2,4,6, \ldots, 2 d\}$. There are $2 d$ gaps and at most $2 d-1$ linear orders in $\mathcal{B}$. It follows that there is some gap $(i, i+1)$ for which there is no integer $j$ with $j \in[s]$ so that $i<S<i+1$ in $L_{j}$. This implies that $q(i, S, \mathcal{B})=q(i+1, S, \mathcal{B})$ so that $\tau(q(i, S, \mathcal{B}))=\tau(q(i+1, S, \mathcal{B}))$. This is a contradiction since one of $i$ and $i+1$ is in $S$ while the other is not.

We comment in closing that Theorem 2.5 can be easily strengthened to yield the following result.

\footnotetext{
${ }^{3}$ This statement does not apply for the definition of Boolean dimension used in [7].
} 
Theorem 2.7. For every $d \geq 1$, there is a constant $c_{d}$ so that if $P$ is poset and every maximal element of $P$ is comparable with at most d elements of $P$, then $\operatorname{bdim}(P) \leq$ $c_{d}$.

Furthermore, we note that Trotter and Walczak [35] proved that if $P$ is a poset and $\lim (P) \leq 3$, then $\operatorname{bdim}(P) \leq 8443$. However, they also proved that for every $d \geq 1$, there is a poset $P$ with $\operatorname{bdim}(P) \geq d$ and $\operatorname{ldim}(P) \leq 4$. Accordingly, in general, neither Boolean dimension nor local dimension is bounded in terms of the other.

\section{Basic Inequalities for Dimension}

Dimension, local dimension and Boolean dimension are all monotonic parameters. But, it is natural to ask whether they are "continuous", i.e., if $Q$ is a subposet of $P$ obtained by removing a single point from $P$, are the values for $Q$ close to the corresponding values for $P$ ?

For dimension, the following elementary result was proved by Hiraguchi [12]. We include a short proof as the basic idea will be important in the discussion to follow.

Theorem 3.1. Let $P$ be a poset on two or more points and let $x$ be an element of $P$. Then $\operatorname{dim}(P) \leq 1+\operatorname{dim}(P-\{x\})$.

Proof. Let $Q=P-\{x\}$, let $d=\operatorname{dim}(Q)$ and let $\left\{L_{1}, L_{2}, \ldots, L_{d}\right\}$ be a realizer of $Q$. For an integer $i \in[d-1]$, let $M_{i}$ be any linear extension of $P$ such that the restriction of $M_{i}$ to $Q$ is $L_{i}$. Let $Y$ be the ground set of $Q$ and let $D(x)$ consist of all points of $Q$ which are less than $x$ in $P$. Dually, let $U(x)$ consist of all points of $Q$ which are greater than $x$ in $P$. Define $M_{d}$ and $M_{d+1}$ by:

$$
\begin{aligned}
M_{d} & =L_{d}(D(x))<x<L_{d}(Y-D(x)) \quad \text { and } \\
M_{d+1} & =L_{d}(Y-U(x))<x<L_{d}(U(x)) .
\end{aligned}
$$

Clearly, $\left\{M_{1}, M_{2}, \ldots, M_{d+1}\right\}$ is a realizer of $P$.

We now prove the analogous inequality for local dimension, although the argument is a bit more complex.

Theorem 3.2. Let $P$ be a poset on two or more points and let $x$ be an element of $P$. Then $\operatorname{ldim}(P) \leq 1+\operatorname{ldim}(P-\{x\})$.

Proof. Let $Q=P-\{x\}$. We show that if $d=\operatorname{ldim}(Q)$, then $\operatorname{ldim}(P) \leq d+1$.

Now let $\mathcal{L}$ be a local realizer of $Q$. Clearly, we may assume that $\mu(y, \mathcal{L})=d$ for every $y \in Q$. Let $y_{0} \in Q$ and relabel the ple's in $\mathcal{L}$ as $\left\{L_{1}, L_{2}, \ldots, L_{t}\right\}$ so that $y_{0} \in L_{i}$ when $i \in[d]$. For each $i \in[d]$, let $Q_{i}$ be the subposet of $P$ determined by the ground set of $L_{i}$. It follows that if $u \in Q$, then $u \in Q_{i}$ for some $i \in[d]$. Then for each $i \in[d]$, let $M_{i}$ be a linear extension of the subposet of $P$ determined by elements of $Q_{i}$ and $x$ for which the restriction of $M_{i}$ to $Q_{i}$ is $L_{i}$.

Let $I(x)=\{u \in P: x \| u$ in $P\}$. If $I(x)=\varnothing$, then

$$
\left\{M_{i}: i \in[d]\right\} \cup\left\{L_{j}: d+1 \leq j \leq t\right\}
$$


is a local realizer for $P$ and this would imply that $\operatorname{ldim}(P)=d$. So we may assume that $I(x) \neq \varnothing$.

Let $W=\left\{w \in I(x): w \notin Q_{d}, x>w\right.$ in $M_{i}$ for all $i \in[d-1]$ with $\left.w \in Q_{i}\right\}$. Also, set $Z=\left\{z \in I(x): z \notin Q_{d}, x<z\right.$ in $M_{i}$ for all $i \in[d-1]$ with $\left.z \in Q_{i}\right\}$. Note that $W \cap Z=\varnothing$.

The ple $L_{d}$ has the block form $A<\{x\}<B$. Then let $A^{\prime}=A \cap I(x)$ and $B^{\prime}=B \cap I(x)$. We then define ple's $N_{1}$ and $N_{2}$ as follows: The ground set of $N_{1}$ is $\{x\} \cup Q_{d} \cup W$ and the ground set of $N_{2}$ is $\{x\} \cup Q_{d} \cup Z$. These two ple's will have the following block form:

$$
\begin{aligned}
& N_{1}=A-A^{\prime}<\{x\}<A^{\prime} \cup B \cup W, \\
& N_{2}=A \cup B^{\prime} \cup Z<\{x\}<B-B^{\prime} .
\end{aligned}
$$

Note that no element in $W$ is less than an element in $A-A^{\prime}$, or else it would be comparable to $x$. The analogous assertion holds for elements in $Z$ and $B-B^{\prime}$. Furthermore, the ordering of elements of $A-A^{\prime}$ in $N_{1}$ is equal to the ordering of $A-A^{\prime}$ in $L_{d}$. A similar assertion holds for elements of $A^{\prime} \cup B$ in $N_{1}, B-B^{\prime}$ in $N_{2}$, and $A \cup B^{\prime}$ in $N_{2}$ when comparing to the ordering in $L_{d}$. It follows that:

$$
\mathcal{L}^{\prime}=\left\{M_{i}: 1 \leq i<d\right\} \cup\left\{L_{j}: d<j \leq t\right\} \cup\left\{N_{1}, N_{2}\right\}
$$

is a local realizer for $P$ with $\mu\left(P, \mathcal{L}^{\prime}\right)=d+1$.

We do not know whether the analogous result holds for Boolean dimension. In fact, here is the best inequality we have been able to obtain concerning the removal of a single point.

Theorem 3.3. Let $P$ be a poset on two or more points and let $x$ be an element of $P$. Then $\operatorname{bdim}(P) \leq 3+\operatorname{ldim}(P-\{x\})$.

Proof. Let $(\mathcal{B}, \tau)$ be a Boolean realizer for $Q=P-\{x\}$, with $|\mathcal{B}|=\operatorname{bdim}(Q)=d$. Label the linear orders in $\mathcal{B}$ as $\left\{L_{1}, L_{2}, \ldots, L_{d}\right\}$. For each $i \in[d]$, let $M_{i}$ be the linear order on the ground set of $P$ defined by setting $M_{i}=x<L_{i}$. Next, we set $M_{d+1}=x<L_{1}^{*}$.

Now let $L$ be any linear extension of $P$. With a shift in subscripts and letting $Y$ be the ground set of $Q$, we follow the proof of Theorem 3.1 and set:

$$
\begin{aligned}
& M_{d+2}=L(D(x))<x<L(Y-D(x)) \quad \text { and } \\
& M_{d+3}=L(Y-U(x))<x<L(U(x)) .
\end{aligned}
$$

Note that $M_{d+2}$ and $M_{d+3}$ are linear extensions of $P$.

Then set $\hat{\mathcal{B}}=\left\{M_{1}, M_{2}, \ldots, M_{d+3}\right\}$. For a pair $(u, v)$ of distinct points of $P$, we claim that we can always determine whether $u$ is less than $v$ in $P$ based on the bits in the string $q(u, v, \hat{\mathcal{B}})$. First, we consider the bits associated with the linear orders in $\left\{M_{1}, M_{2}, \ldots, M_{d}, M_{d+1}\right\}$. If one of $u$ and $v$ is $x$, these bits are constant; otherwise they are not. Furthermore, if one of $u$ and $v$ is $x$, we can tell whether $u<v$ in $P$ from the bits associated with the linear extensions $M_{d+2}$ and $M_{d+3}$. If neither $u$ nor $v$ is $x$, then we can tell whether $u$ is less than $v$ in $P$ by applying $\tau$ to the bits associated with $\left\{M_{1}, M_{2}, \ldots, M_{d}\right\}$. 


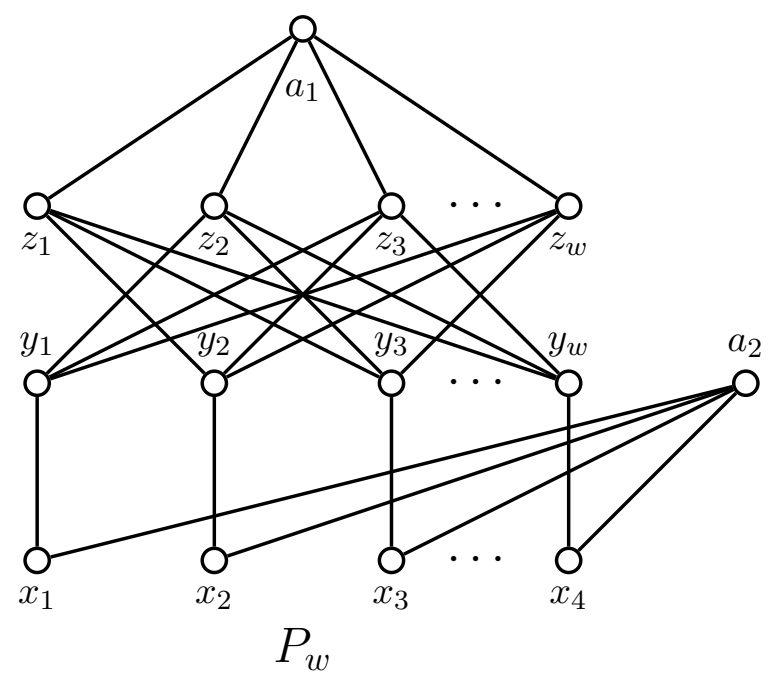

Figure 1. Showing the Inequality is Tight

3.1. Inequalities involving Width. In his classic 1950 paper [4, Dilworth observed in a first page footnote that an immediate consequence of his chain partitioning theorem is that the Dushnik-Miller dimension of a poset is at most its width. The standard examples show that this elementary inequality is best possible. To date, we have not been able to determine the maximum local dimension of a poset of width $w(w \geq 4)$. While it is bounded above by $w$, we do not know if this is a tight upper bound. The analogous question for Boolean dimension also remains open.

Although it may seem surprising, we have been able to settle analogous questions for more complex inequalities involving width. As one such example, the following inequality was proved by Trotter [32].

Theorem 3.4. Let $P$ be a poset and let $A=\operatorname{Max}(P)$. If $P-A$ is non-empty and has width $w$, then $\operatorname{dim}(P) \leq w+1$ and this is sharp.

In [32, a family $\left\{P_{w}: w \geq 2\right\}$ of posets is constructed to show that the inequality in Theorem 3.4 is tight for Dushnik-Miller dimension. These posets are shown in Figure 1.

As noted in [32], it is straightforward to verify that for each $w \geq 3$, the poset $P_{w}$ is $(w+1)$-irreducible. However, it is an easy exercise to show that all the posets in this family have local dimension at most 4 , and they have Boolean dimension at most 4 . Therefore it remains to answer the following: Is the inequality in Theorem 3.4 tight for local dimension or for Boolean dimension? We will explain why the answer for both parameters is yes, but we elect to postpone the argument until we have discussed a second inequality involving width.

The following inequality was also proved in [32].

Theorem 3.5. Let $A$ be an antichain in a poset $P$ with $P-A$ non-empty. If the width of the subposet $P-A$ is $w$, then $\operatorname{dim}(P) \leq 2 w+1$. 
The argument to show that this inequality is best possible is more complex, and a construction to accomplish this task is given by Trotter in a separate paper [31]. We now analyze a "one-sided" variation of that construction.

For a pair $(n, w)$ of positive integers, we define a poset $P=P(n, w)$ containing $n w+n^{w}$ points. The subposet $P-\operatorname{Max}(P)$ contains $n w$ elements that form a disjoint sum of $w$ chains each of size $n$ : $C_{1}+C_{2}+\ldots+C_{w}$. We label the points of $C_{i}$ as $x_{i, 1}<x_{i, 2}<\ldots<x_{i, n}$. For each sequence $\sigma=\left(j_{1}, j_{2}, \ldots, j_{w}\right) \in[n]^{w}$ of positive integers taken from $[n]$, there is a maximal element $a_{\sigma}$ of $P$ with $a_{\sigma}$ covering $x_{i, j_{i}}$ in $P$ for each $i \in[w]$. Note that there are $n^{w}$ maximal elements in $P$, and in the argument below, we will denote the set $\operatorname{Max}(P)$ of maximal elements of $P$ just as $A$.

We also require a special case of a result which has become known as the "Product Ramsey Theorem," given in the classic text [11] as Theorem 5 on page 113. However, we will use slightly different notation in discussing this result.

When $A_{1}, A_{2}, \ldots, A_{t}$ are $k$-element subsets of $B_{1}, B_{2}, \ldots, B_{t}$, respectively, we refer to the Cartesian product $A_{1} \times A_{2} \times \cdots \times A_{t}$ as a $\mathbf{k}^{t}$-grid in $B_{1} \times B_{2} \times \cdots \times B_{t}$. Here is a formal statement of the version of the Product Ramsey Theorem we will use.

Theorem 3.6. Let $(k, t, h, r)$ be a 4-tuple of positive integers with $h \geq k$. There exists a least positive integer $n_{0}=\operatorname{PRam}(k, t, h, r)$ such that if $n \geq n_{0}, g$ is an $\mathbf{n}^{t}$ grid and $\varphi$ is a coloring of all $\mathbf{k}^{t}$-grids in $g$ with $r$ colors, then there exists an $\mathbf{h}^{t}$-grid $g^{\prime}$ in $g$ such that all $\mathbf{k}^{t}$-grids in $g^{\prime}$ are mapped to the same color by $\varphi$.

With these preparatory remarks in hand, here is the result we will prove. This theorem shows that the inequality in Theorem 3.4 is best possible for both local dimension and Boolean dimension.

Theorem 3.7. For every $w \geq 1$, there is an integer $n_{0}$ so that if $n \geq n_{0}$, then $\operatorname{ldim}(P(n, w))=\operatorname{bdim}(P(n, w))=w+1$. Note that $w$ is the width of $P(n, w)-$ $\operatorname{Max}(P(n, w))$.

Proof. We give full details of the proof for local dimension, which is slightly more complicated. At the end, we will outline how an argument for Boolean dimension can be structured.

Since $\operatorname{ldim}(P(n, w) \leq \operatorname{dim}(P(n, w)) \leq w+1$, we need only show that $\operatorname{ldim}(P(n, w)) \geq$ $w+1$, provided $n$ is sufficiently large. This assertion holds trivially when $w=1$, so we will fix a value $w \geq 2$, assume that $\lim (P(n, w)) \leq w$ for all $n$ and argue to a contradiction.

We consider a large, but unspecified value of $n$, and we let $\mathcal{L}=\left\{L_{i}: i \in[t]\right\}$ be a local realizer of $P(n, w)$ with $\mu(P, \mathcal{L}) \leq w$. Clearly, we may assume $\mu(z, \mathcal{L})=w$ for every $z \in P(n, w)$.

Next, we describe a coloring $\varphi$ of the $\mathbf{2}^{w}$ grids in $C_{1} \times C_{2} \times \cdots \times C_{w}$. For each $i \in[w]$, consider a 2-element subset $S_{i}=\left\{j_{i}, j_{i}^{\prime}\right\}$ of $C_{i}$ with $j_{i}<j_{i}^{\prime}$. Note that $g=S_{1} \times S_{2} \times \cdots \times S_{w}$ is a $\mathbf{2}^{w}$ grid. With the grid $g$, we associate the antichain $\left\{x_{i, j_{i}^{\prime}}: i \in[w]\right\}$ and an element $a(g)$ of $\operatorname{Max}(P)$. We set $a(g)=a_{\sigma}$, where $\sigma=\left(j_{1}, j_{2}, \ldots, j_{w}\right)$. Clearly, $a(g)$ is incomparable with each element of the antichain. Therefore for each $i \in[w]$, there is a least positive integer $m_{i} \in[t]$ so that $a(g)<x_{i, j_{i}^{\prime}}$ 
in $L_{m_{i}}$. Then we set $\varphi(g)=\left(\left(\alpha_{1}, \beta_{1}\right),\left(\alpha_{2}, \beta_{2}\right), \ldots,\left(\alpha_{w}, \beta_{w}\right)\right)$, where occurrence $\alpha_{i}$ of $a(g)$ and occurrence $\beta_{i}$ of $x_{i, j_{i}^{\prime}}$ is in $L_{m_{i}}$, for each $i \in[w]$.

The number of colors used by $\varphi$ is $w^{2 w}$, thus we take $n \geq \operatorname{PRam}\left(5, w, 2, w^{2 w}\right)$. Theorem 3.6 implies that there exists a $5^{w}$-grid $H_{1} \times H_{2} \times \ldots \times H_{w}$ such that every $\mathbf{2}^{w}$-grid within it is assigned the same color:

$$
\left(\left(\alpha_{1}, \beta_{1}\right),\left(\alpha_{2}, \beta_{2}\right), \ldots,\left(\alpha_{w}, \beta_{w}\right)\right) .
$$

We relabel the elements of $P$ so that $H_{i}=\left\{x_{i, 1}, x_{i, 2}, x_{i, 3}, x_{i, 4}, x_{i, 5}\right\}$ with $x_{i, 1}<x_{i, 2}<$ $x_{i, 3}<x_{i, 4}<x_{i, 5}$, for each $i \in[w]$.

Consider the $\mathbf{2}^{w}$-grids of the form $g_{a}=S_{1} \times S_{2} \times \cdots \times S_{w}$ where $S_{i}=\left\{x_{i, 1}, x_{i, a}\right\}$ with $a \in\{2,3,4,5\}$, for each $i \in[w]$. These grids show that there is a sequence $\left(m_{1}, m_{2}, \ldots, m_{w}\right)$ of not necessarily distinct integers so that for each $i \in[w]$, occurrence $\beta_{i}$ of $x_{i, 2}, x_{i, 3}, x_{i, 4}$ and $x_{i, 5}$ all occur in $L_{m_{i}}$.

Let us show that the elements of the sequence $\left(m_{1}, m_{2}, \ldots, m_{w}\right)$ are pairwise distinct. Suppose, for a contradiction, that $m_{1}=m_{2}$, noting that this argument can be applied for the case where any other two elements of the sequence are equal. Let $S_{i}=\left\{x_{i, 4}, x_{i, 5}\right\}$ for $i \in[w]$ and consider the $\mathbf{2}^{w}$-grids $g_{1}=\left\{x_{1,1}, x_{1,2}\right\} \times S_{2} \times S_{3} \times$ $\ldots \times S_{w}$, and $g_{2}=S_{1} \times\left\{x_{2,1}, x_{2,2}\right\} \times S_{3} \times \ldots \times S_{w}$. We must have $a\left(g_{1}\right)<x_{1,4}$ in $L_{m_{1}}$, and $a\left(g_{2}\right)<x_{2,2}$ in $L_{m_{2}}$. As $m_{1}=m_{2}$ this implies $a\left(g_{1}\right), x_{1,4}, a\left(g_{2}\right)$, and $x_{2,2}$ appear in $L_{m_{1}}$. Since $x_{1,4}<a\left(g_{2}\right)$ in $P$, it follows that $a\left(g_{1}\right)<x_{1,4}<a\left(g_{2}\right)<x_{2,2}$ in $L_{m_{1}}$. This is not possible as $x_{2,2}<a\left(g_{1}\right)$ in $P$. Therefore the integers in the sequence $\left(m_{1}, m_{2}, \ldots, m_{w}\right)$ are all distinct.

Now let $\sigma=(2,2,2, \ldots, 2)$ and $\sigma^{\prime}=(3,3,3, \ldots, 3)$. It follows that $x_{i, 2}<a_{\sigma}<$ $x_{i, 3}<a_{\sigma^{\prime}}<x_{i, 4}$ in $L_{m_{i}}$ for each $i \in[w]$. This accounts for all $w$ of the occurrences of $a_{\sigma}$ and $a_{\sigma^{\prime}}$. As a consequence, there is no ple $L$ in $\mathcal{L}$ with $a_{\sigma}>a_{\sigma^{\prime}}$ in $L$. Since $a_{\sigma}$ is incomparable to $a_{\sigma^{\prime}}$ in $P$ this implies that $\mathcal{L}$ is not a local realizer for $P$. The contradiction completes the proof of the theorem for local dimension.

Here is an outline of the argument for Boolean dimension. As before, suppose that $P=P(n, w)$ has a Boolean realizer $(\mathcal{B}, \tau)$ with $|\mathcal{B}|=w$ and argue to a contradiction when $n$ is sufficiently large. First, use the Product Ramsey theorem to assume that, after relabeling of the chains in $P$ and the linear orders in $\mathcal{B}$, we have the following two properties:

(1) for each $(i, j) \in[w] \times[w]$, the elements of $C_{i}$ appear as a block in $L_{j}$, and

(2) for each $a \in \operatorname{Max}(P)$, and for each $i \in[w]$, if $a$ covers a point $x \in C_{i}$ and $x<x^{\prime}$ in $C_{i}$, then $a$ is between $x$ and $x^{\prime}$ in $L_{i}$.

Once this structure has been identified, it is easy to see that for every bit-string $\sigma$ of length $w$, there is some pair $\left(a, a^{\prime}\right)$ of distinct maximal elements such that $q\left(a, a^{\prime}, \mathcal{B}\right)=\sigma$. Clearly, this results in a contradiction if we simply choose $\sigma$ such that $\tau(\sigma)=1$.

The original construction given in 31 has an antichain $A$ with $n$ chains $C_{1}+$ $C_{2}+\cdots+C_{n}$ below $A$ and $n$ chains $D_{1}+D_{2}+\cdots+D_{n}$ above $A$. Now the size of $A$ is $n^{2 w}$, where each element in $A$ covers exactly one element from each $C_{i}$ and is covered by exactly one element from each $D_{j}, i, j \in[n]$. Using this construction, it 
is straightforward to modify the argument given above to show that the inequality in Theorem 3.5 is best possible for both local dimension and Boolean dimension.

3.2. Dimension and Size. The following well known inequality is due to Hiraguchi [12].

Theorem 3.8. If $n \geq 2$ and $|P| \leq 2 n+1$, then $\operatorname{dim}(P) \leq n$.

The family of standard examples shows that the preceding theorem is best possible for Dushnik-Miller dimension. Accordingly, it is of interest to determine (or at least estimate) the maximum value of the Boolean dimension and the maximum value of the local dimension of a poset on $n$ points.

Resolving this question for Boolean dimension is the principal result in Nešetřil and Pudlak's 1989 paper [28].

Theorem 3.9. The maximum value of the Boolean dimension of a poset on $n$ points is $\Theta(\log n)$.

The lower bound for the preceding theorem results from a simple counting argument. Consider an integer $n=2 m$ and the posets on $2 m$ points with $\left\{a_{1}, a_{2}, \ldots, a_{m}\right\} \subseteq$ $\operatorname{Min}(P)$ and $\left\{b_{1}, b_{2}, \ldots, b_{m}\right\} \subseteq \operatorname{Max}(P)$. Clearly, there are $2^{m^{2}}$ such posets. If they all have Boolean dimension at most $d$, then we must have

$$
(2 m !)^{d} 2^{2^{d}} \geq 2^{m^{2}}
$$

This implies that $d=\Omega(\log n)$. The argument given in 28] to show that the maximum Boolean dimension of a poset on $n$ points is $O(\log n)$ is more complex.

Quite recently, Kim, Martin, Masařík, Shull, Smith, Uzzell and Wang [20] have settled the analogous question for local dimension using clever probabilistic methods. Both upper and lower bounds of their proof are non-trivial.

Theorem 3.10. The maximum value of the local dimension of a poset on $n$ points is $\Theta(n / \log n)$.

3.3. Dimension and the Complement of Antichains. The following inequality was proved independently by Trotter [32] and Kimble [21].

Theorem 3.11. Let $A$ be an antichain in a poset $P$ and let $n=|P-A|$. Then $\operatorname{dim}(P) \leq \max \{2, n\}$.

The standard examples again show that the inequality in Theorem 3.11 is best possible. Moreover, this inequality coupled with the fact that $\operatorname{dim}(P)$ is at most the width of $P$ yields a simple proof of Hiraguchi's inequality.

For local dimension we have the following analogue, a result where Theorem $\underline{3.10}$ plays an important role.

Theorem 3.12. The maximum value of the local dimension of a poset $P$ consisting of an antichain $A$ and $n$ other points is $\Theta(n / \log n)$.

Proof. The argument for the lower bound in Theorem 3.10 results from considering height 2 posets with $n$ minimal elements and $n$ maximal elements and showing that 
among them, there is (at least) one whose local dimension is $\Omega(n / \log n)$. Accordingly, the same lower bound applies in this theorem as well.

The upper bound is a bit more complicated 4 , and we find it convenient to prove a slightly stronger result, i.e., we show that the local dimension of a poset $P$ is $O(n / \log n)$ when the ground set of $P$ can be partitioned as $A \cup X \cup Y$ where

(1) $A$ is a maximal antichain in $P$;

(2) each point of $X$ is less than some point in $A$;

(3) each point of $Y$ is greater than some point in $A$; and

(4) $|X|=|Y|=n$.

We now build a local realizer $\mathcal{L}=\mathcal{L}_{1} \cup \mathcal{L}_{2} \cup \mathcal{L}_{3} \cup \mathcal{L}_{4}$ of $P$. We start by setting $\mathcal{L}_{1}=\left\{L_{1}, L_{2}\right\}$ where $X<A<Y$ in $L_{1}, X<A<Y$ in $L_{2}$ and the restriction of $L_{1}$ to $A$ is the dual of the restriction of $L_{2}$ to $A$. Using Theorem 3.10, we take the family $\mathcal{L}_{2}$ to be a local realizer of the subposet $Q$ determined by $X \cup Y$ with $\mu\left(u, \mathcal{L}_{2}\right)$ being $O(n / \log n)$ for each point $u \in Q$.

Next, we construct a family $\mathcal{L}_{3}$ of ple's of $X \cup A$ so that

(1) for each incomparable pair $(x, a)$ with $x \in X$ and $a \in A$, there is some $L \in \mathcal{L}_{3}$ with $x>a$ in $L$; and

(2) $\mu\left(u, \mathcal{L}_{3}\right)$ is $O(n / \log n)$ for each $u \in X \cup A$.

We begin by taking an arbitrary partition of $X$ as $X=X_{1} \cup X_{2} \cup \cdots \cup X_{s}$ where each subposet $X_{i}$ has size $m=n / s$. As usual in arguments of this type, we are assuming $s$ and $m$ are integers. For each $i \in[s]$, we let $\mathcal{U}_{i}$ denote the family of all upsets of $X_{i}$. Considering $\mathcal{U}_{i}$ as partially ordered by inclusion, it is clear that $\mathcal{U}_{i}$ can be partitioned into at most $\left(\begin{array}{c}m \\ {[m / 2\rceil}\end{array}\right)$ chains, as $\mathcal{U}_{i}$ is a subposet of the Boolean lattice (or subset lattice).

Now let $S_{1} \subsetneq S_{2} \subsetneq S_{3} \subsetneq \cdots \subsetneq S_{r}$ be any chain in this partition of $\mathcal{U}_{i}$. We build a ple $L$ using the following recursion. Set $D_{1}=S_{1}$ and let $D_{i}=S_{i}-S_{i-1}$ for $2 \leq i \leq r$. An element $x \in X$ will be in $L$ if and only if $x \in S_{r}$. Second, we have $x>y$ in $L$ if there are integers $i$ and $j$ with $1 \leq i<j \leq r$ so that $x \in D_{i}$ and $y \in D_{j}$. The order $L$ assigns to a pair $x, y \in S_{r}$ when there is some $i$ for which $x, y \in D_{i}$ is arbitrary.

To complete the definition of $L$ we add those elements $a \in A$ such that there is some $i$ for which $a$ is incomparable with all elements of $S_{i}$ and comparable with all elements of $X_{i}-S_{i}$. Of course, we place $a$ immediately under the lowest element of $S_{i}$ in $L$.

Now we count frequencies. Each element of $X$ is in a unique subposet $X_{i}$. So, being generous $\mu\left(x, \mathcal{L}_{3}\right) \leq\left(\begin{array}{c}m \\ {[m / 2\rceil}\end{array}\right)$. On the other hand, for each $i \in[s]$, an element $a \in A$ appears in at most one ple associated with chains in the partition of $\mathcal{U}_{i}$. It follows that $\mu\left(a, \mathcal{L}_{3}\right) \leq s$.

So to optimize the construction, we choose $s$ so that $s=\left(\begin{array}{c}m \\ m / 2\end{array}\right)$. This yields that $\mu\left(u, \mathcal{L}_{3}\right)$ is $O(n / \log n)$ for every $u \in X \cup A$.

To complete the proof, the preceding construction is then repeated in a symmetric manner to obtain a family $\mathcal{L}_{4}$ for $Y \cup A$.

\footnotetext{
${ }^{4}$ This part of the proof is a result of conversations in 2016 with S. Felsner, P. Micek and V. Wiechert.
} 
For Boolean dimension, we have been able to show that there is a constant $C$ such that $\operatorname{bdim}(P) \leq\lceil 2 n / 3\rceil+C$ when $P$ contains an antichain $A$ and $n$ other points. We do not include the details as we feel the result is most likely far from best possible.

3.4. Dimension and the Product of Chains. For positive integers $k$ and $d$, let $\mathbf{k}^{d}$ denote the Cartesian product of $d$ copies of a $k$-element chain. As is well known, for all $k \geq 2, \operatorname{dim}\left(\mathbf{k}^{d}\right)=d$. It is an easy application of the Product Ramsey Theorem to show that for each $d \geq 1$, there is an integer $k_{d}$ so that if $k \geq k_{d}$, then $\operatorname{bdim}\left(\mathbf{k}^{d}\right)=$ $\operatorname{ldim}\left(\mathbf{k}^{d}\right)=d$. However, we are completely unable to settle whether or not $k_{d}=2$ when $d \geq 2$. An easy counting $\operatorname{argument}$ shows that $\operatorname{bdim}\left(\mathbf{2}^{d}\right)=\Omega(d / \log d)$, but it might be the case that $\operatorname{bdim}\left(\mathbf{2}^{d}\right)=d$. We know even less about the situation with local dimension.

3.5. Components and Blocks. We assume that the reader is familiar with basic concepts of graph theory, including the following terms: connected and disconnected graphs, components, cut vertices and $k$-connected graphs for an integer $k \geq 2$. Recall that when $G$ is a graph, a connected induced subgraph $H$ of $G$ is called a block of $G$ when $H$ is a maximal subgraph with no cut vertex.

Here are the analogous concepts for posets. A poset $P$ is said to be connected if its cover graph is connected. A subposet $B$ of $P$ is said to be convex if $y \in B$ whenever $x, z \in B$ and $x<y<z$ in $P$. Note that when $B$ is a convex subposet of $P$, the cover graph of $B$ is an induced subgraph of the cover graph of $P$. A convex subposet $B$ of $P$ is called a component of $P$ when the cover graph of $B$ is a component of the cover graph of $P$. A convex subposet $B$ of $P$ is called a block of $P$, when the cover graph of $B$ is a block in the cover graph of $P$.

As is well known, when $P$ is a disconnected poset with components $C_{1}, C_{2}, \ldots, C_{t}$, for some $t \geq 2, \operatorname{dim}(P)=\max \left(\{2\} \cup\left\{\operatorname{dim}\left(C_{i}\right): 1 \leq i \leq t\right\}\right)$. Readers may note that the preceding observation is just a special case of the formula for the dimension of a lexicographic sum (see page 23 in [33]). For local dimension, it is an easy exercise to show that $\lim (P) \leq 2+\max \left\{\operatorname{ldim}\left(C_{i}\right): 1 \leq i \leq t\right\}$, but we do not know whether this inequality is best possible.

The corresponding result for Boolean dimension is more complicated and is due to Mészáros, Micek and Trotter [25].

Theorem 3.13. Let $P$ be a disconnected poset with components $C_{1}, C_{2}, \ldots, C_{t}$, for some $t \geq 2$. If $d=\max \left\{\operatorname{bdim}\left(C_{i}\right): 1 \leq i \leq t\right\}$, then $\operatorname{bdim}(P) \leq 2+d+4 \cdot 2^{d}$.

The inequality in Theorem 3.13 cannot be improved dramatically, since it is shown in [25] that for large $d$, there is a disconnected poset $P$ with $\operatorname{bdim}(P)=\Omega\left(2^{d} / d\right)$ and $\operatorname{bdim}(C) \leq d$ for every component $C$ of $P$.

The situation with blocks is more complex, even for Dushnik-Miller dimension. In [38], Trotter, Walczak and Wang prove the following result for Dushnik-Miller dimension.

Theorem 3.14. If $d \geq 1$ and $\operatorname{dim}(B) \leq d$ for every block of a poset $P$, then $\operatorname{dim}(P) \leq d+2$. Furthermore, this inequality is best possible. 
Neither the proof of the inequality in Theorem 3.14, nor the proof that the inequality is best possible is elementary. Surprisingly, however, there is no analogous result for local dimension, as Bosek, Grytczuk and Trotter [3] prove that for every $d \geq 4$, there is a poset $P$ with $\operatorname{ldim}(P) \geq d$, such that $\operatorname{ldim}(B) \leq 3$ whenever $B$ is a block in $P$.

However, on the issue of blocks, Boolean dimension behaves like Dushnik-Miller dimension, as the following inequality is proved in [25].

Theorem 3.15. If $d \geq 1$ and $\operatorname{bdim}(B) \leq d$ for every block $B$ of a poset $P$, then $\operatorname{bdim}(P) \leq 9+d+18 \cdot 2^{d}$.

Again, this inequality cannot be improved dramatically, as it is shown in [25] that for large $d$, there is a poset $P$ with $\operatorname{bdim}(P)=\Omega\left(2^{d} / d\right)$ and $\operatorname{bdim}(B) \leq d$ for every block $B$ of $P$.

\section{Planar Posets and Dimension}

A poset $P$ is planar if its order diagram can be drawn in the plane without edge crossings. If a poset is planar, then its cover graph is planar, although the converse does not hold in general. It is easy to see that the standard example $S_{n}$ is planar when $2 \leq n \leq 4$ and non-planar when $n \geq 5$.

In Figure 2, we show a construction due to Kelly [19] showing that for all $n \geq 5$, the non-planar poset $S_{n}$ is a subposet of a planar poset. This specific figure is a diagram where $n=5$, but it should be clear how we intend that the diagram should be modified for other values of $n$. Of course, the Kelly posets show that there are planar posets with arbitrarily large dimension.

In retrospect, the Kelly posets should have prompted research on the following questions:

(1) Must a planar poset with large dimension have large height?

(2) Must a planar poset with large dimension have many minimal elements (and many maximal elements)?

(3) Must a planar poset with large dimension contain two large chains with all points in one incomparable with all points in the other?

(4) Must a planar poset with large dimension contain a large standard example?

However, these natural questions lay dormant for more than 20 years, so here is a compact summary of work done in the last five years. The first three questions in this listing have been answered in the affirmative. However, the last question in the list has been open for nearly 30 years.

In 2014, Streib and Trotter [30] proved that for every positive integer $h$, there is a least positive integer $c_{h}$ so that if $P$ is a poset of height $h$ and the cover graph of $P$ is planar, then $\operatorname{dim}(P) \leq c_{h}$. The proof given in [30] merely established the existence of $c_{h}$ and gave no useful information about its size. However, an exponential upper bound was given in [16], and more recently, two groups have announced a polynomial upper bound on $c_{h}$. Joret, Micek, Ossona de Mendez and Wiechert have shown how their results in [16] can be extended to obtain this conclusion. Meanwhile, Kozik, Krawczyk, Micek and Trotter 24] have a much more complicated argument which 


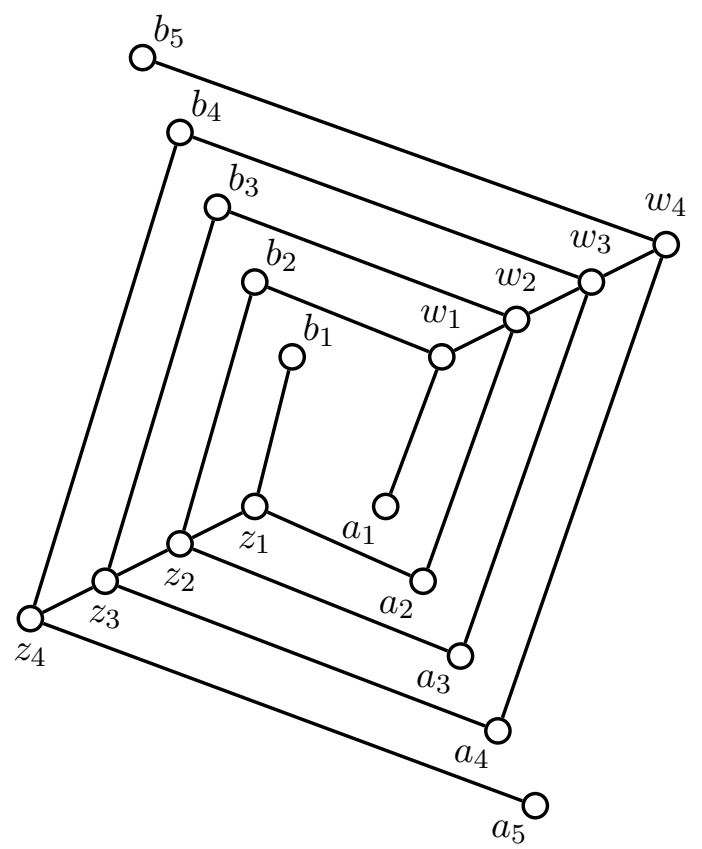

Figure 2. The Kelly Construction

yields a better exponent. From Below, Joret, Micek and Wiechert [17] showed that the $c_{h} \geq 2 h-2$.

For planar posets, Joret, Micek and Wiechert [17] have a linear upper bound, i.e., they show that a planar poset of height $h$ has dimension at most $192 h+96$. They have also given $4 h / 3-2$ as a lower bound.

In [36], Trotter and Wang proved that the dimension of a planar poset with $t$ minimal elements is at most $2 t+1$. They also showed that this inequality is tight for $t=1$ and $t=2$. For $t \geq 3$, they were only able to show that there is a planar poset with $t$ minimal elements which has dimension $t+3$. Using duality, analogous statements hold for maximal elements. Note, however, that there are no statements of this type for posets with planar cover graphs, since as pointed out in [30, for every $d \geq 1$, there is a poset $P$ with a zero and a one such that $\operatorname{dim}(P) \geq d$ and the cover graph of $P$ is planar.

In [14], Howard, Streib, Trotter, Walczak and Wang proved that for each $k \geq 1$, there is a constant $d_{k}$ so that if $P$ is a poset which does not contain two chains $C_{1}$ and $C_{2}$ each of size $k$ such that all points of $C_{1}$ are incomparable with all points of $C_{2}$, then the dimension of $P$ is at most $d_{k}$.

In [8], Felsner, Trotter and Wiechert showed that if $P$ is a poset and the cover graph of $P$ is outerplanar, then $\operatorname{dim}(P) \leq 4$. They also gave an example to show that the inequality is best possible. This same example shows that the inequality is tight for Boolean dimension and local dimension. The argument for Boolean dimension 
is trivial, while the argument for local dimension has the same spirit as the proof of Theorem 2.1. We leave the details of this proof as an exercise.

In [28], Nešetřil and Pudlák note that the Kelly posets have Boolean dimension at most 4, and they asked whether Boolean dimension is bounded for the class of planar posets. This challenging question remains open. We note that it is conceivable (although we consider it very unlikely) that Boolean dimension is bounded for planar posets but unbounded for posets with planar cover graphs.

In presenting his concept of local dimension to conference participants, Ueckerdt noted that standard examples have local dimension at most 3, and it is easy to see that in fact, the Kelly posets have local dimension at most 3. This leads naturally to the question: Do planar posets have bounded local dimension? However, this question has recently been answered in the negative by Bosek, Grytczuk and Trotter [3].

\section{Connections with Structural Graph Theory}

In this section, we explore which variants of dimension can be bounded in terms of path-width or tree width. For the sake of completeness, we include here the basic definitions of tree-width and path-width. Let $G$ be a graph with vertex set $V(G)$. A tree-decomposition of $G$ is a pair $(T, \mathcal{B})$ where $T$ is a tree with vertex set $V(T)$, and $\mathcal{B}=\left\{B_{t}: t \in V(T)\right\}$ is a family of subsets of $V(G)$ satisfying:

$\left(T_{1}\right)$ for each $v \in V(G)$ there exists $t \in V(T)$ with $v \in B_{t}$; and for every edge $u v$ in $G$ there exists $t \in V(T)$ with $u, v \in B_{t}$;

$\left(T_{2}\right)$ for each $v \in V(G)$, if $v \in B_{t} \cap B_{t^{\prime \prime}}$ for some $t, t^{\prime \prime} \in V(T)$, and $t^{\prime}$ lies on the path in $T$ between $t$ and $t^{\prime \prime}$, then $v \in B_{t^{\prime}}$.

It is common to refer to the tree $T$ as the host tree in the tree-decomposition, and when $t \in V(T)$, the induced subgraph $G\left[B_{t}\right]$ of $G$ is referred to as a bag. Note that, $\left|B_{t}\right|$ is just the number of vertices of $G\left[B_{t}\right]$.

The width of a tree-decomposition $(T, \mathcal{B})$ is defined as

$$
\max _{t \in V(T)}\left\{\left|B_{t}\right|-1\right\}
$$

The tree-width of $G, \operatorname{tw}(G)$, is the minimum width of a tree-decomposition of $G$.

A tree-decomposition $\left(T,\left\{B_{t}: t \in V(T)\right\}\right)$ is called a path-decomposition when the host tree $T$ is a path. In turn, the path-width of $G, \operatorname{pw}(G)$, is the minimum width of a path-decomposition of $G$. Observe that $\operatorname{pw}(G) \geq \operatorname{tw}(G)$ since every path-decomposition of $G$ is a tree-decomposition of $G$.

We encourage readers to consult the discussion of connections between DushnikMiller dimension and structural graph theory as detailed in [15], 36] and [35]. Here we provide a quick summary of highlights.

The first major result linking dimension and structural graph theory is due to Joret, Micek, Milans, Trotter, Walczak and Wang [15, who proved that the dimension of a poset is bounded as a function of its height and the tree-width of its cover graph. More formally, they showed that for each pair $(t, h)$ of positive integers, there is a least positive integer $d(t, h)$ so that if $P$ is a poset of height $h$ and the 
tree-width of the cover graph of $P$ is $t$, then $\operatorname{dim}(P) \leq d(t, h)$. A poset of height 1 is an antichain and has dimension at most 2 , so it is of interest to study $d(t, h)$ only when $h \geq 2$. Trotter and Moore [34] showed that $d(1, h)=3$ for all $h \geq 2$, and Joret, Micek, Trotter, Wang, and Wiechert [18] showed that $d(2, h) \leq 1276$ for all $h \geq 2$. As is well known, Kelly posets have cover graphs with path-width at most 3 , so $d(t, h)$ goes to infinity with $h$ when $t \geq 3$.

Joret, Micek and Wiechert [17] have recently shown that for fixed $t \geq 3, d(t, h)$ grows exponentially with $h$. The best bound to date in the general case is due to Joret, Micek, Ossona de Mendez and Wiechert [16], where they prove:

$$
\left.2^{\Omega(h\lfloor(t-1) / 2\rfloor}\right) \leq d(t, h) \leq 4^{\left(\begin{array}{c}
t+3 h-3 \\
t
\end{array}\right)} .
$$

Now we turn our attention to analogous results for Boolean dimension and local dimension. In 2016, Micek and Walczak 26] proved that the Boolean dimension of a poset is bounded in terms of the path-width of its cover graph, independent of its height. In 2017, Felsner, Mészáros and Micek [7 proved that in fact, the Boolean dimension of a poset is bounded in terms of the tree-width of its cover graph, independent of its height.

Now on to local dimension. We will first prove the following result which asserts that the local dimension of a poset is bounded in terms of the path-width of its cover graph, independent of its height.

Theorem 5.1. For every $t \geq 1$, there is a least positive integer $d(t)$ so that if $P$ is a poset whose cover graph has path-width $t$, then $\operatorname{ldim}(P) \leq d(t)$.

The details of the proof show that $d(t)$ is $O\left(5^{(t+1)^{2}}\right)$. However, we will then show that the local dimension of a poset is not bounded in terms of the tree-width of its cover graph independent of its height.

Theorem 5.2. For every $d \geq 1$, there exists a poset $P$ with $\operatorname{ldim}(P)>d$ such that the cover graph of $P$ has tree-width at most 3 .

5.1. Local Dimension and Path-Width. Here we give the proof of Theorem 5.1, Our argument requires some preliminary material on a concept introduced by Kimble [22]. The split of a poset $P$ is the height 2 poset $Q$ whose minimal elements are $\left\{x^{\prime}: x \in P\right\}$ and whose maximal elements are $\left\{x^{\prime \prime}: x \in P\right\}$. Furthermore, for all $x, y \in P$ not necessarily distinct, $x^{\prime}<y^{\prime \prime}$ in $Q$ if and only if $x \leq y$ in $P$.

The following well known result is an easy exercise, but it is stated here for emphasis.

Theorem 5.3. Let $Q$ be the split of a poset $P$. Then $\operatorname{dim}(P) \leq \operatorname{dim}(Q) \leq 1+$ $\operatorname{dim}(P)$.

Recent work in dimension theory has made use of a variant of the notion of a split. Let $P$ be a poset and let $X$ denote the ground set of $P$. The split-in-place of $P$ is the poset $R$ obtained as follows:

(1) The ground set of $R$ is disjoint union of three sets $X \cup X^{\prime} \cup X^{\prime \prime}$.

(2) $X^{\prime}=\left\{x^{\prime}: x \in X\right\}=\operatorname{Min}(R)$ and $X^{\prime \prime}=\left\{x^{\prime \prime}: x \in X\right\}=\operatorname{Max}(R)$. 
(3) The subposet of $R$ determined by $X$ is $P$.

(4) In $R$, for each $x \in X, x^{\prime}$ is only covered by $x$, and $x^{\prime \prime}$ only covers $x$.

Observe that the split of $P$ is a subposet of the split-in-place of $P$.

Essentially the same argument used to prove Theorem 5.3 yields the following extension.

Theorem 5.4. Let $Q$ be the split and let $R$ be the split-in-place of a poset $P$. Then $\operatorname{dim}(P) \leq \operatorname{dim}(Q) \leq \operatorname{dim}(R) \leq 1+\operatorname{dim}(P)$.

We note that there is no analogue of this theorem for Boolean dimension. Indeed, while the Boolean dimension of the canonical interval order is unbounded, it is easy to show that the split of any interval order has Boolean dimension at most 6 . Here is the analogue of the preceding theorem for local dimension.

Lemma 5.5. Let $Q$ be the split and let $R$ be the split-in-place of a poset $P$. Then $\operatorname{ldim}(P) \leq \operatorname{ldim}(R) \leq 2 \operatorname{ldim}(Q)-1$ and $\operatorname{ldim}(Q) \leq \operatorname{ldim}(R) \leq 2+\operatorname{ldim}(P)$.

Proof. The inequalities $\operatorname{ldim}(P) \leq \operatorname{ldim}(R)$ and $\operatorname{ldim}(Q) \leq \operatorname{ldim}(R)$ hold since both $P$ and $Q$ are subposets of $R$.

Setting $s=\operatorname{ldim}(Q)$, we show that $\operatorname{ldim}(R) \leq 2 s-1$. Let $\mathcal{L}=\left\{L_{1}, L_{2}, \ldots, L_{t}\right\}$ be a local realizer of $Q$ with $\mu(u, \mathcal{L})=s$ for every $u \in Q$. Recall that the ground set of $R$ is $X \cup X^{\prime} \cup X^{\prime \prime}$, where $X$ is the ground set of $P$. For each $i \in[t]$, let $X_{i}^{\prime}$ consist of those elements $x^{\prime} \in X^{\prime}$ which are in $L_{i}$ and let $X_{i}^{\prime \prime}$ consist of those elements $x^{\prime \prime} \in X^{\prime \prime}$ which are in $L_{i}$. Then let $X_{i}$ consist of those elements $x \in X$ for which either $x^{\prime} \in X_{i}^{\prime}$ or $x^{\prime \prime} \in X_{i}^{\prime \prime}$.

For the poset $R$, let $M_{i}$ be a ple whose ground set is $X_{i} \cup X_{i}^{\prime} \cup X_{i}^{\prime \prime}$ such that the restriction of $M_{i}$ to $X_{i}^{\prime} \cup X_{i}^{\prime \prime}$ is $L_{i}$. Checking the necessary details it can be seen that $\mathcal{M}=\left\{M_{1}, M_{2}, \ldots, M_{t}\right\}$ is a local realizer of $R$, with $\mu(R, \mathcal{M}) \leq 2 s$. However, since for each $x \in P$ there is some $i$ with $x^{\prime}<x^{\prime \prime}$ in $L_{i}$ it follows that $\mu(R, \mathcal{M}) \leq 2 s-1$.

Next, we show that $\operatorname{ldim}(R) \leq 2+\operatorname{ldim}(P)$. Let $d=\operatorname{ldim}(P)$ and let $\mathcal{L}=$ $\left\{L_{1}, L_{2}, \ldots, L_{t}\right\}$ be a local realizer of $P$ with $\mu(x, \mathcal{L})=d$ for all $x \in P$. For each $i \in[t]$, let $X_{i}$ be the ground set of the ple $L_{i}$. We then modify $L_{i}$ as follows: for each $x \in X_{i}$, we add $x^{\prime}$ immediately under $x$ and we add $x^{\prime \prime}$ immediately over $x$.

It remains to witness the incomparabilities $(a, b)$ where both $a$ and $b$ are in $X^{\prime}$ or both are in in $X^{\prime \prime}$. Construct two linear extensions of $R$ as follows. Let $L$ be a linear extension of $P$. Take $M_{0}$ to be the linear extension of $R$ with block structure $X^{\prime}<X<X^{\prime \prime}$, where the restriction to each of $X, X^{\prime}$ and $X^{\prime \prime}$ is ordered according to the corresponding elements in $L$. Similarly, define $M_{0}^{\prime}$ to be the linear extension of $R$ with block structure $\bar{X}^{\prime}<X<\bar{X}^{\prime \prime}$, where $\bar{X}^{\prime}$ and $\bar{X}^{\prime \prime}$ are ordered dually to $L$, and $X$ is ordered according to $L$. Now, we can see that $\mathcal{M}=\left\{M_{0}, M_{0}^{\prime}\right\} \cup\left\{M_{i}: i \in[t]\right\}$ is a local realizer for $R$ with $\mu(R, \mathcal{M}) \leq 2+d$.

For the remainder of the proof, we fix a positive integer $t$ and let $P$ be a poset whose cover graph has path-width at most $t$. We will then show that $\operatorname{ldim}(P)$ is $O\left(5^{(t+1)^{2}}\right)$. Let $Q$ be the split of $P$, and let $R$ be the split-in-place of $P$. The basic idea for the remainder of the argument is to prove that the path-width of the cover 
graph of $R$ is $t+1$. We will use this to show that the local dimension of $Q$ is bounded in terms of $t$. The conclusion of our theorem will then follow from Lemma 5.5.

We let $\mathbb{P}_{n}$ denote the path whose vertex set is $[n]$ with vertices $i$ and $j$ from $[n]$ adjacent in $\mathbb{P}_{n}$ if and only if $|i-j|=1$.

Then let $G$ be the cover graph of $P$ and let $H$ be the cover graph of $R$.

Let $\mathcal{P}_{G}=\left(\mathbb{P}_{m},\left\{B_{t}: t \in[m]\right\}\right)$ be a path-decomposition of $G$ of width $t$. If $u \in P$ we define $a_{\mathcal{P}_{G}}(u)=\min \left\{t \in[m]: u \in B_{t}\right\}$ and $b_{\mathcal{P}_{G}}(u)=\max \left\{t \in[m]: u \in B_{t}\right\}$. Since $\mathcal{P}_{G}$ is a path-decomposition, it follows that $u \in B_{t}$ if and only if $a_{\mathcal{P}_{G}}(u) \leq t \leq$ $b_{\mathcal{P}_{G}}(u)$. Thus we define the interval of $u$ in $\mathcal{P}_{G}$ to be the set of consecutive integers $\operatorname{Int}_{\mathcal{P}_{G}}(u)=\left\{t \in[m]: a_{\mathcal{P}_{G}}(u) \leq t \leq b_{\mathcal{P}_{G}}(u)\right\}$. We may assume that the endpoints of the intervals in $\mathcal{P}_{G}$ are distinct and every bag contains an endpoint, i.e., for each $i \in[m]$, there is exactly one vertex $u \in P$ with $i \in\left\{a_{\mathcal{P}_{G}}(u), b_{\mathcal{P}_{G}}(u)\right\}$.

Let us fix the path-decomposition for $H$ to be $\mathcal{P}=\left(\mathbb{P}_{3 m},\left\{B_{t}^{\prime}: t \in[3 m]\right\}\right)$, where

$$
B_{3 i-j}^{\prime}= \begin{cases}B_{i} \cup\left\{u^{\prime \prime}\right\} & \text { if } j=0 \text { and } i=a_{\mathcal{P}_{G}}(u) \text { for some } u \in P, \\ B_{i} \cup\left\{u^{\prime}\right\} & \text { if } j=1 \text { and } i=a_{\mathcal{P}_{G}}(u) \text { for some } u \in P, \\ B_{i} & \text { if } j=2 \text { and } i=a_{\mathcal{P}_{G}}(u) \text { for some } u \in P, \\ B_{i} & \text { if } j \in\{0,1,2\} \text { and } i \neq a_{\mathcal{P}_{G}(u)} \text { for all } u \in P .\end{cases}
$$

Since the path-decomposition of $H$ is now fixed we let $n=3 m$, and we adopt similar notation for intervals in $\mathcal{P}$ as done above. That is, if $u \in R$, then $a_{u}=\min \{t \in[n]$ : $\left.u \in B_{t}^{\prime}\right\}, b_{u}=\max \left\{t \in[n]: u \in B_{t}^{\prime}\right\}$, and $\operatorname{Int}(u)=\left\{t \in[n]: a_{u} \leq t \leq b_{u}\right\}$. Note that $\mathcal{P}$ has width $t+1$ and that it satisfies the following properties:

(1) The endpoints of the intervals in $\mathcal{P}$ are distinct, i.e., for each $i \in[n]$, there is at most one vertex $u \in R$ with $i \in\left\{a_{u}, b_{u}\right\}$.

(2) For every $u \in P, b_{u^{\prime}}<a_{u^{\prime \prime}}$.

(3) For every pair $(u, v)$ of (not necessarily distinct) elements of $P$, if $\operatorname{Int}(v)$ intersects either of $\operatorname{Int}\left(u^{\prime}\right)$ and $\operatorname{Int}\left(u^{\prime \prime}\right)$, then it contains both of them.

We now begin to use properties of $\mathcal{P}$ to build a local realizer $\mathcal{L}$ for $Q$. To avoid a proliferation of primes and double primes in the presentation, we will adopt the following conventions: the letter $x$, sometimes written with subscripts, will always denote a minimal element of $Q$. Dually, the letter $y$ will always denote a maximal element of $Q$. Also, we take $X$ as the set of all minimal elements of $Q$ while $Y$ is the set of all maximal elements of $Q$. We let $\operatorname{Inc}(X, Y)$ denote the set of all pairs $(x, y) \in X \times Y$ with $(x, y) \in \operatorname{Inc}(Q)$.

We begin by including two linear extensions $L_{0}$ and $L_{0}^{\prime}$ in $\mathcal{L}$ such that for all $(x, y) \in X \times Y, x<y$ in both $L_{0}$ and $L_{0}^{\prime}$, in which:

(1) the restriction of $L_{0}$ to $X$ is the dual of the restriction of $L_{0}^{\prime}$ to $X$, and

(2) the restriction of $L_{0}$ to $Y$ is the dual of the restriction of $L_{0}^{\prime}$ to $Y$.

Given a set of ple's $\mathcal{L}^{\prime}$ which satisfy the following condition, then $\mathcal{L}=\mathcal{L}^{\prime} \cup\left\{L_{0}, L_{0}^{\prime}\right\}$ is a local realizer for $Q$.

Reversing Min-Max Pairs. For each pair $(x, y) \in \operatorname{Inc}(X, Y)$, there is some $L \in \mathcal{L}^{\prime}$ with $x>y$ in $L$. 
Of course, we must take care to keep $\mu(z, \mathcal{L})$ bounded in terms of $t$ for all $z \in Q$. We begin by taking $\varphi$ as a proper coloring of the graph $G$ in the sense that for each pair of distinct vertices $u, v \in P$ we have $\varphi(u) \neq \varphi(v)$ when $\operatorname{Int}(u) \cap \operatorname{Int}(v) \neq \varnothing$. Let us see why such a coloring exists using $t+1$ colors. Let $\mathcal{P}^{\prime}$ be the path-decomposition of $G$ resulting from restricting the bags of $\mathcal{P}$ to only contain elements of $G$. Consider the ordering of $V(G)$ given by the left endpoints of intervals in $\mathcal{P}^{\prime}$. We may greedily color vertices of $G$ according to this ordering. Since $\mathcal{P}^{\prime}$ witnesses that $\operatorname{pw}(G) \leq t$, the resulting coloring does not use more than $t+1$ colors. Without loss of generality, we may assume that $\varphi$ uses the integers in $[t+1]$ as colors.

Second, for each $z \in X \cup Y$, we let $\operatorname{stack}(z)$ denote the set of all points $u \in P$ such that $\operatorname{Int}(z) \subseteq \operatorname{Int}(u)$. Note that $|\operatorname{stack}(z)| \leq t+1$. We then define a coloring $\pi$ of the elements of $X \cup Y$. The colors used by $\pi$ are vectors of length $t+1$ and the coordinates are taken from $\{0,1,2,3\}$, so $\pi$ uses $4^{t+1}$ colors. Below $\pi(z)(i)$ is the $i$-th coordinate in the $(t+1)$-tuple of $\pi(z)$. Note that there is at most one $u \in \operatorname{stack}(z)$ with $\varphi(u)=i$, because $\varphi$ is a proper coloring (by definition of $\varphi$ ). For each $z \in X \cup Y$ and each $i \in[t+1]$, we set:

(0) $\pi(z)(i)=0$ if there is no element $u \in \operatorname{stack}(z)$ with $\varphi(u)=i$.

(1) $\pi(z)(i)=1$ if there is an element $u \in \operatorname{stack}(z)$ with $\varphi(u)=i$ and $z<u$ in $R$.

(2) $\pi(z)(i)=2$ if there is an element $u \in \operatorname{stack}(z)$ with $\varphi(u)=i$ and $z>u$ in $R$.

(3) $\pi(z)(i)=3$ if there is an element $u \in \operatorname{stack}(z)$ with $\varphi(u)=i$ and $z \| u$ in $R$.

Next, we define a coloring $\tau$ of ordered pairs of elements from $X \cup Y$. The colors used by $\tau$ are $(t+1) \times(t+1)$ matrices with all entries taken from $\{0,1,2,3,4\}$, so $\tau$ uses $5^{(t+1)^{2}}$ colors. For each pair $(z, w)$ of elements of $X \cup Y$ and each pair $(i, j) \in[t+1]^{2}$, we set:

(0) $\tau(z, w)(i, j)=0$ if there is no pair $(u, w)$ with $u \in \operatorname{stack}(z), v \in \operatorname{stack}(w)$, $\varphi(u)=i$ and $\varphi(v)=j$.

(1) $\tau(z, w)(i, j)=1$ if there is a pair $(u, w)$ with $u \in \operatorname{stack}(z), v \in \operatorname{stack}(w)$, $\varphi(u)=i, \varphi(v)=j$ and $u<v$ in $P$.

(2) $\tau(z, w)(i, j)=2$ if there is a pair $(u, w)$ with $u \in \operatorname{stack}(z), v \in \operatorname{stack}(w)$, $\varphi(u)=i, \varphi(v)=j$ and $u>v$ in $P$.

(3) $\tau(z, w)(i, j)=3$ if there is a pair $(u, w)$ with $u \in \operatorname{stack}(z), v \in \operatorname{stack}(w)$, $\varphi(u)=i, \varphi(v)=j$ and $u \| v$ in $P$.

(4) $\tau(z, w)(i, j)=4$ if there is a pair $(u, w)$ with $u \in \operatorname{stack}(z), v \in \operatorname{stack}(w)$, $\varphi(u)=i, \varphi(v)=j$ and $u=v$ in $P$.

Let $x, y \in R$. We say $x$ is left of $y$ if and only if $b_{x}<a_{y}$. Under the same conditions, we say $y$ is right of $x$. Now define a coloring $\sigma$ of the pairs in $\operatorname{Inc}(X, Y)$ using 4 -tuples of the form $\left(\alpha_{1}, \alpha_{2}, \alpha_{3}, \alpha_{4}\right)$. The first coordinate $\alpha_{1}$ is 0 if $x$ is left of $y$ and 1 if $x$ is right of $y$. The remaining three coordinates are defined by setting $\alpha_{2}=\pi(x), \alpha_{3}=\pi(y)$ and $\alpha_{4}=\tau(x, y)$. Clearly, $\sigma$ uses $2 \cdot 4^{2(t+1)} \cdot 5^{(t+1)^{2}}$ colors.

Since the number of colors used by $\sigma$ is bounded in terms of $t$, to complete the proof, it suffices to show that for each color $\Gamma=\left(\alpha_{1}, \alpha_{2}, \alpha_{3}, \alpha_{4}\right)$ used by $\sigma$, we 
can determine a family $\mathcal{L}(\Gamma)$ of ple's so that $(1)$ For each $(x, y) \in \operatorname{Inc}(X, Y)$ with $\sigma(x, y)=\Gamma$, there is some $L \in \mathcal{L}(\Gamma)$ with $x>y$ in $L$; and (2) For each $z \in X \cup Y$, $\mu(z, \mathcal{L}(\Gamma))$ is bounded in terms of $t$.

Fix a color $\Gamma=\left(\alpha_{1}, \alpha_{2}, \alpha_{3}, \alpha_{4}\right)$ and consider the subset $S(\Gamma)$ of $\operatorname{Inc}(X, Y)$ consisting of all pairs $(x, y) \in \operatorname{Inc}(X, Y)$ with $\sigma(x, y)=\Gamma$. We will assume that $\alpha_{1}=0$, i.e., if $(x, y) \in S(\Gamma)$ then all our pairs will have $x$ left of $y$. From the details of the argument, it will be clear that the case $\alpha_{1}=1$ is symmetric. Of course, we will also assume that the set $S(\Gamma)$ is non-empty.

The next part of the proof will involve four claims. We begin by proving the following.

Claim 1. Let $x_{1}, x_{2} \in X$ and $y_{1}, y_{2} \in Y$. Then the following two statements hold:

(1) If $\left(x_{1}, y_{1}\right)$ and $\left(x_{2}, y_{1}\right)$ are in $S(\Gamma)$, and $y_{1}$ is left of $y_{2}$, then $\tau\left(x_{1}, y_{2}\right)=$ $\tau\left(x_{2}, y_{2}\right)$. In particular, $\left(x_{1}, y_{2}\right) \in S(\Gamma)$ if and only if $\left(x_{2}, y_{2}\right) \in S(\Gamma)$.

(2) If $\left(x_{2}, y_{1}\right)$ and $\left(x_{2}, y_{2}\right)$ are in $S(\Gamma)$, and $x_{1}$ is left of $x_{2}$, then $\tau\left(x_{1}, y_{1}\right)=$ $\tau\left(x_{1}, y_{2}\right)$. In particular, $\left(x_{1}, y_{1}\right) \in S(\Gamma)$ if and only if $\left(x_{1}, y_{2}\right) \in S(\Gamma)$.

Proof. Let us prove (1), noting that the proof for (2) follows from a similar argument. Suppose $\tau\left(x_{1}, y_{2}\right)(i, j)=k$ for some $(i, j) \in[t+1]^{2}$ and $k \in\{0,1,2,3,4\}$. We show that $\tau\left(x_{2}, y_{2}\right)(i, j)=k$. This results in the following five cases

- Assume that $k=0$. If $\pi\left(y_{2}\right)(j)=0$, then $\tau\left(x_{2}, y_{2}\right)=0$. However, if $\pi\left(y_{2}\right)(j) \neq 0$ then $\pi\left(x_{1}\right)(i)=0$. Since $\sigma\left(x_{1}, y_{1}\right)=\sigma\left(x_{2}, y_{1}\right)$ this implies in particular that $\pi\left(x_{1}\right)(i)=\pi\left(x_{2}\right)(i)$. So $\pi\left(x_{2}\right)(i)=0$ and therefore $\tau\left(x_{2}, y_{2}\right)=0$.

- If $k=4$, then $i=j$ and there exists $u \in \operatorname{stack}\left(x_{1}\right) \cap \operatorname{stack}\left(y_{2}\right)$. Since $x_{1}$ is left of $y_{1}$ and $y_{1}$ is left of $y_{2}$, then $u \in \operatorname{stack}\left(y_{1}\right)$. Therefore $\tau\left(x_{1}, y_{1}\right)(i, i)=4$. This implies $\tau\left(x_{2}, y_{1}\right)(i, i)=4$, as $\sigma\left(x_{1}, y_{1}\right)=\sigma\left(x_{2}, y_{1}\right)$. Thus $u \in \operatorname{stack}\left(x_{2}\right)$ and it follows that $\tau\left(x_{2}, y_{2}\right)(i, i)=4$.

- For the case where $k=1$, there is $u \in \operatorname{stack}\left(x_{1}\right)$ and $v \in \operatorname{stack}\left(y_{2}\right) \operatorname{such}$ that $\varphi(u)=i, \varphi(v)=j$, and $u<v$ in $P$. Let $u=u_{1} u_{2} \ldots u_{m}=v$ be a path in $G$ that witnesses the comparability $u<v$. It follows that $u_{l} \in \operatorname{stack}\left(y_{1}\right)$ for some $l \in[m]$. Suppose $\varphi\left(u_{l}\right)=j^{\prime}$. Let us assume that $\tau\left(x_{1}, y_{1}\right)\left(i, j^{\prime}\right)=1$, noting that the case where $\tau\left(x_{1}, y_{1}\right)\left(i, j^{\prime}\right)=4$ follows from an analogous argument. Since $\tau\left(x_{1}, y_{1}\right)\left(i, j^{\prime}\right)=1$, this is witnessed by $u \in \operatorname{stack}\left(x_{1}\right)$ and $u_{l} \in \operatorname{stack}\left(y_{1}\right)$. Since $\sigma\left(x_{1}, y_{1}\right)=\sigma\left(x_{2}, y_{1}\right)$ and $\tau\left(x_{1}, y_{1}\right)\left(i, j^{\prime}\right)=1$, we conclude that $\tau\left(x_{2}, y_{1}\right)\left(i, j^{\prime}\right)=1$. Therefore there is $u^{\prime} \in \operatorname{stack}\left(x_{2}\right)$ with $\varphi\left(u^{\prime}\right)=i$ and $u^{\prime}<u_{l}$. We now have $u^{\prime}<v$ and therefore $\tau\left(x_{2}, y_{2}\right)(i, j)=1$.

- The case where $k=2$ follows from an argument analogous to the one for $k=1$.

- We have shown that $\tau\left(x_{1}, y_{2}\right) \neq 3$ if and only if $\tau\left(x_{2}, y_{2}\right) \neq 3$. Thus the result holds when $k=3$.

Claim 2. Let $S \subseteq S(\Gamma)$. Then the following two statements hold:

(1) If there is some $z \in X$ such that $(z, y) \in S(\Gamma)$ whenever $(x, y) \in S$, then the set $S$ is reversible. 
(2) If there is some $w \in Y$ such that $(x, w) \in S(\Gamma)$ whenever $(x, y) \in S$, then the set $S$ is reversible.

Proof. We prove the first statement and note that the proof for (2) is symmetric. We argue by contradiction and assume there is some $k \geq 2$ for which there is a strict alternating cycle $\mathcal{S}=\left\{\left(x_{i}, y_{i}\right): i \in[k]\right\}$ contained in $S$. Without loss of generality, we may assume that the pairs of this cycle have been labeled so that $y_{1}$ is left of $y_{i}$ for all $i \in\{2,3, \ldots, k\}$. Note that $\left(x_{i}, y_{i}\right) \in S$ and $x_{i} \| y_{i}$ in $R$ for all $i \in[k]$. Since $\left(z, y_{i}\right) \in S(\Gamma)$, it follows that $z \| y_{i}$ for all $i \in[k]$. Because $\left(x_{1}, y_{1}\right)$ and $\left(z, y_{1}\right)$ are in $S(\Gamma)$ with $y_{1}$ left of $y_{2}$, Claim 1 guarantees $\left(x_{1}, y_{2}\right) \in S(\Gamma)$ since $\left(z, y_{2}\right) \in S(\Gamma)$, thus $x_{1} \| y_{2}$. This is not possible since $x_{1}<y_{2}$ in $\mathcal{S}$.

We consider the pairs in $S(\Gamma)$ as edges in a bipartite graph $G(\Gamma)$ whose vertex set is $X \cup Y$ with vertex $x \in X$ adjacent to vertex $y \in Y$ in $G(\Gamma)$ when $(x, y) \in S(\Gamma)$. In general, the graph $G(\Gamma)$ may be disconnected and some of the components may just be isolated vertices. Regardless, since a vertex from $X \cup Y$ belongs to at most one component of $G(\Gamma)$, it is enough to consider a subset of $S(\Gamma)$ consisting of pairs determining a non-trivial component of $G(\Gamma)$. Let $C$ be such a component, let $S_{C}$ be the edge set of $C$, and let $X_{C}$ and $Y_{C}$ be, respectively, the subsets of $X$ and $Y$ which are incident with at least one edge in $S_{C}$. Also, let $x_{0}$ be the left-most element of $X_{C}$.

Then using the graph-theoretic concept of distance in a connected graph, for each edge $(x, y) \in S_{C}$, we define $\rho(x, y)=\min \left\{\operatorname{dist}\left(x, x_{0}\right)\right.$, dist $\left.\left(y, x_{0}\right)\right\}$ to be the distance from the edge $(x, y)$ to the vertex $x_{0}$. For each non-negative integer $s$, we let $S_{C}(s)$ denote the set of edges $(x, y) \in S_{C}$ with $\rho(x, y)=s$. Note that $S_{C}(0)$ is just the set of edges $\left(x_{0}, y\right) \in S_{C}$ where $y \in Y_{C}$. The set $X_{C}(s)$ consists of all vertices $x \in X_{C}$ incident with an edge in $S_{C}(s)$. The set $Y_{C}(s)$ is defined analogously. It is obvious that for each $x \in X_{C}$, there are at most two values of $s$ for which $x \in X_{C}(s)$. Furthermore, if there are two values, then they are consecutive integers and the smaller of the two is odd. Similarly, if $y \in Y_{C}$, there are at most two values of $s$ for which $y \in Y_{C}(s)$. If there are two values, they are consecutive integers and the smaller of the two is even.

Claim 3. The following two statements hold:

(1) If $s$ is a non-negative even integer, $\left(x_{1}, y\right) \in S_{C}(s)$ and $\left(x_{2}, y\right) \in S_{C}(s+1)$, then $x_{1}$ is left of $x_{2}$.

(2) If $s$ is an odd positive integer, $\left(x, y_{1}\right) \in S_{C}(s)$ and $\left(x, y_{2}\right) \in S_{C}(s+1)$, then $y_{1}$ is right of $y_{2}$.

Proof. First, suppose that $s=0$. Then since $\left(x_{1}, y\right) \in S_{C}(0)$, we know that $x_{1}=x_{0}$ and therefore $x_{1}$ is left of $x_{2}$ since $x_{0}$ was chosen to be the left-most element of $X_{C}$. Now, we argue by contradiction. Let $s$ be the least positive integer for which one of the two statements of the claim fails.

If $s$ is a positive even integer and the claim fails for the pairs $\left(x_{1}, y\right) \in S_{C}(s)$ and $\left(x_{2}, y\right) \in S_{C}(s+1)$, then $x_{2}$ is left of $x_{1}$. Let $\left(x_{1}, y_{1}\right)$ be any edge in $S_{C}$ so that $\rho\left(x_{1}, y_{1}\right)=s-1$. Since the claim holds for $s-1$, we know that $y_{1}$ is right of 
$y$. By Claim 1, we conclude that $\left(x_{2}, y_{1}\right) \in S_{C}$, so $\rho\left(x_{2}, y_{1}\right) \leq s-1$ because $y_{1}$ is at distance $s-1$ from $x_{0}$. Therefore $\rho\left(x_{2}, y\right) \leq s$, which contradicts the fact that $\rho\left(x_{2}, y\right) \in S_{C}(s+1)$. Therefore $s$ is not a positive even integer.

A similar contradiction is reached when $s$ is a positive odd integer, and with this observation, the proof of the claim is complete.

Accordingly, to complete the proof of our theorem, it is enough to show that for each non-negative integer $s$ and component $C$, there is a family $\mathcal{L}_{C}(s)$ of ple's with ground set $X_{C}(S) \cup Y_{C}(S)$ so that

(1) for each $(x, y) \in S_{C}(s)$, there is some $L \in \mathcal{L}_{C}(s)$ with $x>y$ in $L$; and

(2) $\mu\left(x, \mathcal{L}_{C}(s)\right)$ and $\mu\left(y, \mathcal{L}_{C}(s)\right)$ are bounded in terms of $t$, for every $x \in X_{C}(s)$ and every $y \in Y_{C}(s)$.

The case $s=0$ is easy since all the edges in $S_{C}(0)$ are of the form $\left(x_{0}, y\right)$, and clearly the set of such pairs is reversible. Similarly, the case $s=1$ is handled by Claim 2, since it asserts that the set $S_{C}(1)$ is reversible.

Now we fix an integer $s \geq 2$. Suppose first that $s$ is even. For each $x \in X_{C}(s)$, there is a unique right-most point $w \in Y_{C}$ with $(x, w) \in S_{C}(s-1)$. We call $w$ the right-parent of $x$. For each $w \in Y_{C}$, we then let $X_{C}(s, w)$ denote those elements $x \in X_{C}(s)$ for which $w$ is the right-parent of $x$. Clearly, when $w_{1} \neq w_{2}$, the sets $X_{C}\left(s, w_{1}\right)$ and $X_{C}\left(s, w_{2}\right)$ are disjoint.

For a vertex $w \in Y_{C}$ for which $X_{C}(s, w) \neq \varnothing$, we then let $Y_{C}(s, w)$ denote the set of all $y \in Y_{C}(s)$ for which there is some $x \in X_{C}(s, w)$ with $(x, y) \in S_{C}(s)$.

Claim 4. If $w_{1}$ and $w_{2}$ are distinct elements of $Y_{C}$, then $Y_{C}\left(s, w_{1}\right) \cap Y_{C}\left(s, w_{2}\right)=\varnothing$.

Proof. Suppose to the contrary that there is some $y \in Y_{C}\left(s, w_{1}\right) \cap Y_{C}\left(s, w_{2}\right)$. Choose elements $x_{1} \in X_{C}\left(s, w_{1}\right)$ and $x_{2} \in X_{C}\left(s, w_{2}\right)$ such that $\left(x_{1}, y\right),\left(x_{2}, y\right) \in S_{C}(s)$. Without loss of generality, we may assume that $w_{1}$ is left of $w_{2}$. Since $\left(x_{2}, w_{2}\right) \in$ $S_{C}(s-1)$ and $\left(x_{2}, y\right) \in S_{C}(s)$ where $s$ is even, Claim 3 guarantees $y$ is left of $w_{2}$. Then by Claim $1,\left(x_{1}, w_{2}\right) \in S_{C}$. Clearly, $\rho\left(x_{1}, w_{2}\right)$ is either $s-1$ or $s$. However, if $\rho\left(x_{1}, w_{2}\right)=s$, then the pairs $\left(x_{1}, w_{1}\right)$ and $\left(x_{1}, w_{2}\right)$ violate Claim 3. Also, if $\rho\left(x_{1}, w_{2}\right)=s-1, w_{1}$ is not the right parent of $x_{1}$. The contradiction completes the proof of the claim.

For each $w \in Y_{C}$ for which $X_{C}(s, w) \cup Y_{C}(s, w)$ is non-empty, we form a ple $L_{C}(s, w)$ whose ground set is $X_{C}(s, w) \cup Y_{C}(s, w)$. In view of Claim 2, we may assume that $x>y$ in $L_{C}(s, w)$ for every pair $(x, y) \in S_{C}(s)$ with $x \in X_{C}(s, w)$. In view of Claim 4, for each $z \in X_{C} \cup Y_{C}$, there is at most one element $w \in Y_{C}$ for which $z$ is in the ground set of $L_{C}(s, w)$.

The proof when $s$ is a positive odd integer is similar, except we use the obvious notion of a left-parent rather than a right-parent.

Finally, observe that each element of $Q$ appears at most twice when reversing the incomparable pairs in $S(\Gamma)$. Recall that $\mathcal{L}=\mathcal{L}^{\prime} \cup\left\{L_{0}, L_{0}^{\prime}\right\}$, therefore

$$
\operatorname{ldim}(Q) \leq \mu(Q, \mathcal{L}) \leq 2 \cdot 2 \cdot 4^{2(t+1)} \cdot 5^{(t+1)^{2}} .
$$


Now Lemma 5.5 implies that

$$
\lim (P) \leq 2 \cdot 2 \cdot 2 \cdot 4^{2(t+1)} \cdot 5^{(t+1)^{2}}+3 .
$$

Thus $\operatorname{ldim}(P)$ is $O\left(5^{(t+1)^{2}}\right)$, as desired.

5.2. Local Dimension and Tree-Width. We now turn to proving Theorem 5.2 , Recall that our goal is to prove that for every $d \geq 1$, there exists a poset $P$ such that $\lim (P)>d$ and the tree-width of the cover graph of $P$ is at most 3 .

Our argument will require some additional Ramsey theoretic tools. The results we use in the proof of Theorem 5.2 are treated in a more comprehensive manner by Milliken 27 5 . However we will find it convenient to use somewhat different notation and terminology.

For a positive integer $n$, we view the complete binary tree $T_{n}$ as the poset whose elements are the binary strings of length at most $n$, with $x \leq y$ in $T_{n}$ when $x$ is a initial segment in $y$. The empty string, denoted $\varnothing$, is then the zero (least element) of $T_{n}$. For all $n \geq 1, T_{n}$ has $2^{n+1}-1$ elements, $2^{n}$ leaves and height $n+1$. By convention, we take $T_{0}$ as the one-point poset whose only element is the empty string.

When $n \geq 1$ and $x$ is a binary string of length $n$, we will denote coordinate $i$ of $x$ as $x(i)$ and when a string is of modest length, we may write it explicitly, e.g., $x=01001101$. Let $x$ be a string of length $p, y$ be a string of length $m$ and $x<y$ in $T_{n}$. We say $y$ is in the left tree above $x$ when $y(p+1)=0$. Similarly, $y$ is in the right tree above $x$ when $y(p+1)=1$.

Let $n$ and $m$ be integers with $n \geq m \geq 0$, and let $\Lambda$ be a subposet of $T_{n}$. We will say $\Lambda$ is a strong copy of $T_{m}$ when there is a function $f: T_{m} \rightarrow \Lambda$ satisfying the following two requirements:

(1) $f$ is a poset isomorphism, i.e., $f$ is a bijection and for all $x, y \in T_{m}, x \leq y$ in $T_{m}$ if and only if $f(x) \leq f(y)$ in $\Lambda$.

(2) For all $x, y \in T_{m}$ with $x<y$ in $T_{m}, y$ is in the left tree above $x$ in $T_{m}$ if and only if $f(y)$ is in the left tree above $f(x)$ in $T_{n}$.

The following result is the special case of Theorem 2.1 from [27] for binary trees and has also been used in [1. In fact, the application here preceded and motivated the work in [1].

Theorem 5.6. For every triple $(m, p, r)$ of positive integers, with $p \geq m$, there is a least positive integer $n_{0}=\mathrm{BTRam}(m, p, r)$ so that if $n \geq n_{0}$ and $\varphi$ is an $r$-coloring of the strong copies of $T_{m}$ in $T_{n}$, then there is a color $\alpha \in[r]$ and a subposet $\Lambda$ of $T_{n}$ such that $\Lambda$ is a strong copy of $T_{p}$ and $\varphi$ assigns color $\alpha$ to every strong copy of $T_{m}$ contained in $\Lambda$.

We now turn our attention to a construction due to Joret, Micek and Wiechert [17] which was used to show that a poset whose cover graph has bounded tree-width can have dimension that grows exponentially with the height of the poset. Here is their

\footnotetext{
${ }^{5}$ The particular result we need is Theorem 2.1 on page 220 . Note that Milliken credits the result to Halpern, Läuchi, Laver and Pincus and comments on the history of the result.
} 

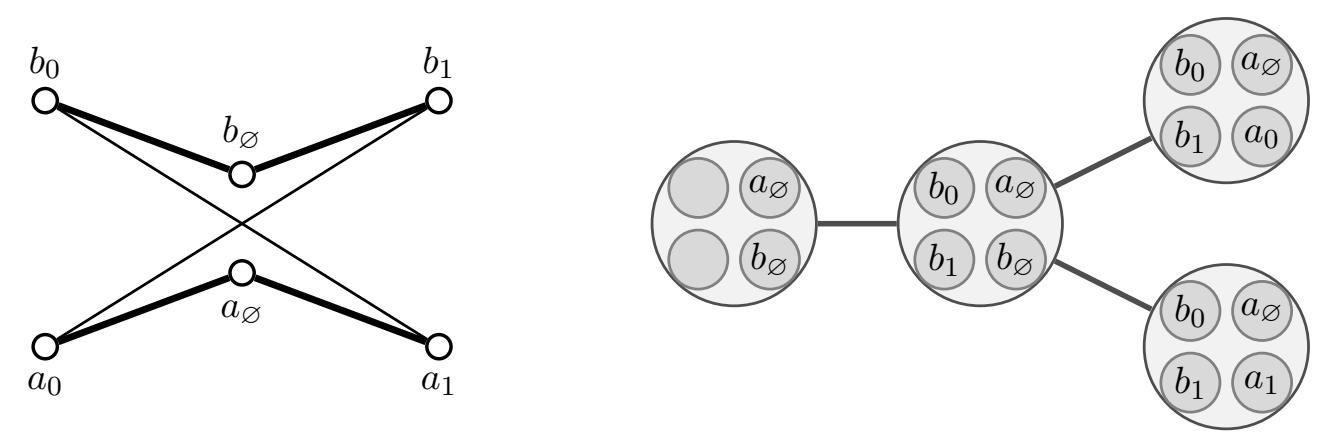

Figure 3. The Joret-Micek-Wiechert Construction for $n=1$

construction, with notation and terminology adjusted so that we can conveniently apply Theorem 5.6.

For $n \geq 0$, construct a poset $P_{n}$ as follows. The ground set of $P_{n}$ is the disjoint union $A_{n} \cup B_{n}$ where $B_{n}$ is an up set and $A_{n}$ is a down set in $P_{n}$. The subposet $B_{n}$ is a copy of $T_{n}$. For each string $x$ in $T_{n}$ of length at most $n$, we let $b_{x}$ be the corresponding element of $B_{n}$, i.e., $b_{x}<b_{y}$ in $B_{n}$ if and only if $x$ is an initial segment of $y$. Note that $b_{\varnothing}$ is the minimum of $B_{n}$.

The subposet $A_{n}$ is a copy of $T_{n}^{*}$, the dual of $T_{n}$. When $x \in T_{n}$, we let $a_{x}$ be the corresponding element of $A_{n}$, i.e., $a_{x}>a_{y}$ in $A_{n}$ if and only if $x$ is an initial segment of $y$. Note that $a_{\varnothing}$ is the maximum of $A_{n}$.

When $\left(a_{x}, b_{y}\right) \in A_{n} \times B_{n}$, we set $a_{x}<b_{y}$ if and only if neither $x$ nor $y$ is an initial segment of the other. For example, $a_{1011}<b_{01}, a_{11}<b_{010}, a_{101} \| b_{10}$ and $a_{101} \| b_{101}$ in $P_{n}$.

In Figure 3 and Figure 4, we show a tree-decomposition of the cover graph of $P_{1}$ and $P_{2}$ that have width 3 respectively. Observe that $P_{1}$ is isomorphic to the standard example $S_{3}$. Also, note that there is a leaf of the host trees in which the only two vertices of $P_{1}$ and $P_{2}$ which occur in this bag are $a_{\varnothing}$ and $b_{\varnothing}$.

Now it is an easy exercise to verify by induction the following properties of the family $\left\{P_{n}: n \geq 0\right\}$ :

(1) The tree-width of the cover graph of $P_{n}$ is (at most) 3, and

(2) $P_{n}$ has a tree-decomposition of width 3 for which there is a leaf (bag) $u$ in the host tree for which the set of elements of $P_{n}$ appearing in $u$ is precisely $\left\{a_{\varnothing}, b_{\varnothing}\right\}$.

To complete the proof of Theorem [5.2, we now prove the following claim.

Claim. Let $d \geq 2$. If $n \geq \operatorname{BTRam}\left(1,3, d^{2}\right)$, then $\operatorname{ldim}\left(P_{n}\right)>d$.

Proof. Let $d \geq 2$ and $n \geq \operatorname{BTRam}\left(1,3, d^{2}\right)$. We assume $\operatorname{ldim}\left(P_{n}\right) \leq d$ and argue to a contradiction. Let $\mathcal{L}=\left\{L_{1}, L_{2}, \ldots, L_{t}\right\}$ be a local realizer for $P_{n}$ with $\mu(\mathcal{L}) \leq d$. We use $\mathcal{L}$ to construct a coloring $\varphi$ of the strong copies of $T_{1}$ in $T_{n}$. A strong copy of $T_{1}$ consists of three binary strings $x, y, z$ with $x$ an initial segment of both $y$ and $z$ and, if $x$ is a string of length $s, y(s+1)=0$ while $z(s+1)=1$. In particular, this implies that $a_{x} \| b_{z}$ in $P_{n}$. 

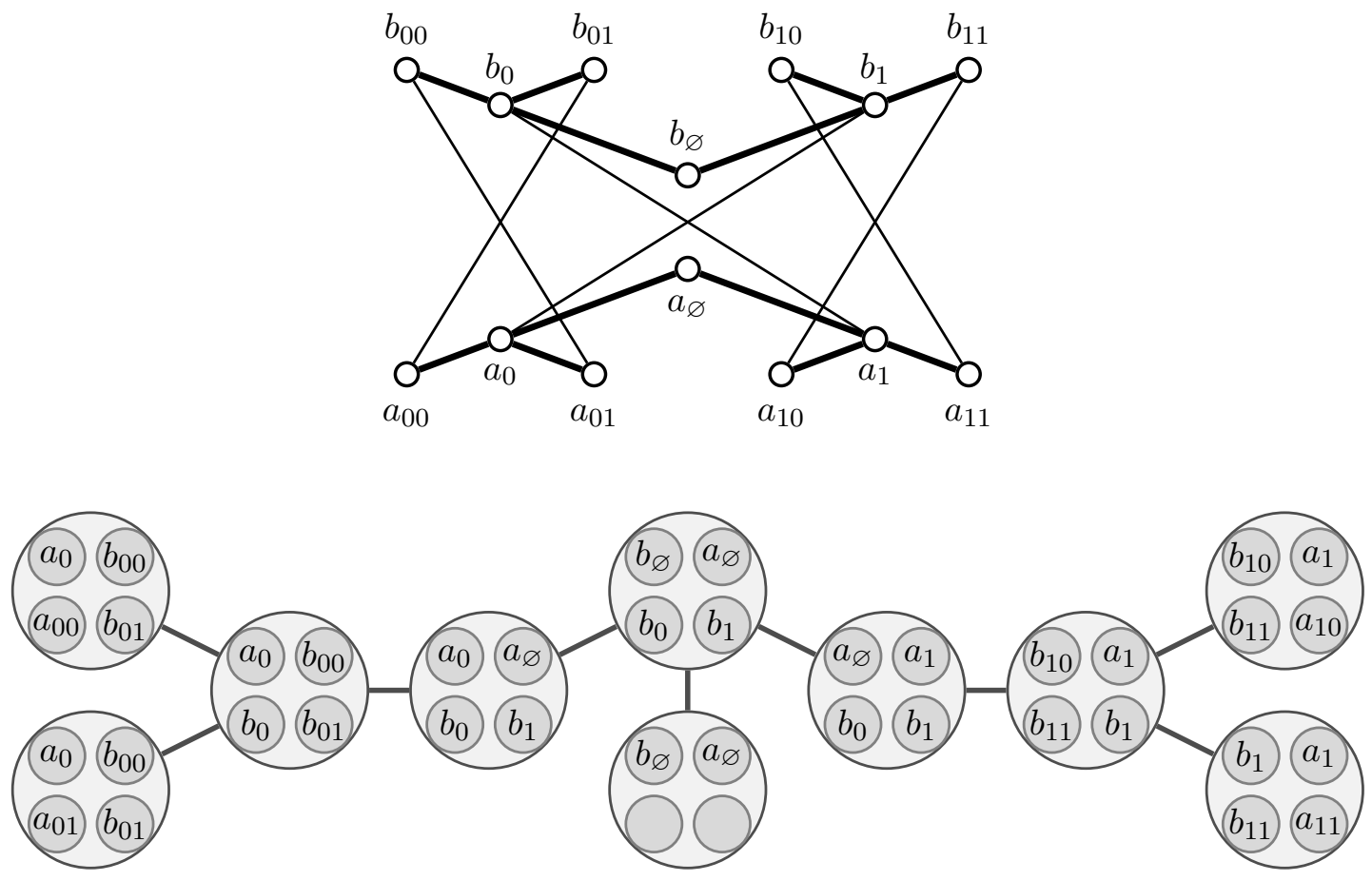

FiguRE 4. The Joret-Micek-Wiechert construction for $n=2$

We then define a $d^{2}$-coloring of the strong copies of $T_{1}$ in $T_{n}$ by setting $\varphi(\{x, y, z\})=$ $(\alpha, \beta)$ where $\alpha$ and $\beta$ are defined as follows: Let $i$ be the least positive integer for which $a_{x}>b_{z}$ in $L_{i}$. Then $(\alpha, \beta)$ is the pair for which occurrence $\alpha$ of $a_{x}$ is in $L_{i}$ and occurrence $\beta$ of $b_{z}$ is in $L_{i}$. Since $\alpha, \beta \in[d]$, the function $\varphi$ uses at most $d^{2}$ colors. We pause to note that the element $y$ plays no role in this argument. Everything to follow depends only on $x$ and $z$.

From Theorem [5.6, there is a subtree $\Lambda$ of $T_{n}$ and a color $(\alpha, \beta)$ so that $\Lambda$ is a strong copy of $T_{3}$ and $\varphi$ maps every strong copy of $T_{1}$ in $\Lambda$ to color $(\alpha, \beta)$. Relabel the elements of $\Lambda$ so that they match the standard notation for $T_{3}$.

Now consider the 3 -element subset $\{\varnothing, 0,111\}$ in $T_{3}$, which is a strong copy of $T_{1}$. This copy is assigned color $(\alpha, \beta)$ so there is some $L_{i} \in \mathcal{L}$ so that $a_{\varnothing}>b_{111}$ in $L_{i}$, where occurrence $\alpha$ of $a_{\varnothing}$ is in $L_{i}$ and occurrence $\beta$ of $b_{111}$ is in $L_{i}$. Next consider the 3 -element subset $\{\varnothing, 0,101\}$ which is also a strong copy of $T_{1}$. Since we already know that occurrence $\alpha$ of $a_{\varnothing}$ is in $L_{i}$, it follows that occurrence $\beta$ of $b_{101}$ is also in $L_{i}$.

We then consider the subsets $\{10,100,101\}$ and $\{11,110,111\}$. Both are strong copies of $T_{1}$. Since we already know that occurrence $\beta$ of $b_{101}$ and occurrence $\beta$ of $b_{111}$ is in $L_{i}$, we conclude that $a_{10}>b_{101}$ in $L_{i}$ and $a_{11}>b_{111}$ in $L_{i}$. This is impossible since $a_{10}<b_{111}$ in $P_{n}$ and $a_{11}<b_{101}$ in $P_{n}$. The contradiction completes the proof. 


\section{Summary Listing of Open Problems}

For the convenience of readers, we gather here a listing of open problems concerning Boolean dimension and local dimension.

(1) For a positive integer $w$, what is the maximum value of the Boolean dimension of a poset whose width is $w$ ?

(2) For a positive integer $w$, what is the maximum value of the local dimension of a poset whose width is $w$ ?

(3) For a non-negative integer $n$, what is the maximum value of the Boolean dimension of a poset consisting of an antichain and $n$ additional points?

(4) Is there a constant $d_{0}$ such that every planar poset has Boolean dimension at most $d_{0}$ ?

(5) Is there a constant $d_{0}$ such that every poset with a planar cover graph has Boolean dimension at most $d_{0}$ ?

(6) If a planar poset has large dimension, must it contain a large standard example?

(7) If a planar poset has large Boolean dimension, must it contain a large standard example?

(8) If a planar poset has large local dimension, must it contain a large standard example?

(9) For an integer $d \geq 4$, what is the maximum local dimension of a disconnected poset in which each component has local dimension at most $d$ ? Note. The answer is either $d, d+1$ or $d+2$.

(10) What is the maximum amount the Boolean dimension of a poset can drop when a single point is removed? Note. The answer is either 1,2 or 3 .

(11) What is the Boolean dimension and the local dimension of $\mathbf{2}^{d}$ ?

\section{ACKnowledgments}

Our work has benefited considerably through collaboration, and a touch of competition, with our colleagues Stefan Felsner, Gwenaël Joret, Tamás Mészáros, Piotr Micek and Bartosz Walczak. Smith was supported in part by NSF-DMS grant 1344199.

\section{REFERENCES}

[1] F. Barrera-Cruz, S. Felsner, T. Mészáros, P. Micek, H. Smith, L. Taylor and W. T. Trotter, Separating Tree-chromatic number from Path-chromatic Number, J. Combin. Theory Ser. B, In press. Available on-line 2019.

[2] C. Biró, P. Hamburger, A. Pór and W. T. Trotter, Forcing posets with large dimension to contain large standard examples, Graphs Combin., 32 (2016), 861-880.

[3] B. Bosek, J. Grytczuk and W. T. Trotter, Local dimension is unbounded for planar posets, preliminary manuscript.

[4] R. P. Dilworth, A decomposition theorem for partially ordered sets, Ann. Math. (2), 41 (1950), 161-166.

[5] B. Dushnik, Concerning a certain set of arrangements, Proc. Amer. Math. Soc. 1 (1950), 788-796.

[6] B. Dushnik and E. W. Miller, Partially ordered sets, Amer. J. Math., 63 (1941), 600-610. 
[7] S. Felsner, T. Mészáros and P. Micek, Boolean dimension and Tree-width, https://arxiv.org/abs/1707.06114.

[8] S. Felsner, W. T. Trotter and V. Wiechert, The dimension of posets with planar cover graphs, Graphs Combin., 31 (2015), 927-939.

[9] G. Gambosi, J. Nešetřil and M. Talamo, Posets, Boolean representations and quick path searching, in Automata, Languages and Programming, 14th International Colloquium, ICALP87, Proceedings, Lecture Note Series in Computer Science, 267, (1987), 404-424.

[10] G. Gambosi, J. Nešetřil and M. Talamo, On locally presented posets, Theoret. Comput. Sci., 70 (1990), 251-260.

[11] R. L. Graham, B. L. Rothschild and J. H. Spencer, Ramsey Theory, 2nd Edition, J. H. Wiley, New York, 1990.

[12] T. Hiraguchi, On the dimension of orders, Sci. Rep. Kanazawa Univ., 4 (1955), 1-20.

[13] S. Hoşten and W. D. Morris, The dimension of the complete graph, Discrete Math., 201 (1998), 133-139.

[14] D. M. Howard, N. Streib, W. T. Trotter, B. Walczak and R. Wang, Dimension of posets with planar cover graphs excluding two long incomparable chains, J. Combin. Theory Ser. A, 164 (2019), 1-23.

[15] G. Joret, P. Micek, K. Milans, W. T. Trotter, B. Walczak and R. Wang, Tree-width and dimension, Combinatorica 36 (2016), 431-450.

[16] G. Joret, P. Micek, P. Ossona de Mendez and V. Wiechert, Nowhere Dense Graph Classes and Dimension, https://arxiv.org/abs/1708.05424

[17] G. Joret, P. Micek and V. Wiechert, Planar posets have dimension at most linear in their height, SIAM J. Discrete Math., 34(1) (2017), 2754-2790.

[18] G. Joret, P. Micek, W. T. Trotter, R. Wang and V. Wiechert, On the dimension of posets with cover graphs of tree-width 2, Order, 34 (2017), 185-234.

[19] D. Kelly, On the dimension of partially ordered sets, Discrete Math., 35 (1981), 135-156.

[20] J. Kim, R. R. Martin, T. Masařík, W. Shull, H. Smith, A. Uzzell and Z. Wang, On difference graphs and the local dimension of posets, https://arxiv.org/abs/1803.08641.

[21] R. J. Kimble, Extremal Problems in Dimension Theory for Partially Ordered Sets, Ph.D. Thesis, Massachusetts Institute of Technology, 1973.

[22] R. J. Kimble, personal communication.

[23] D. J. Kleitman and G. Markovsky, On Dedekind's problem: The number of isotone Boolean functions, II, Trans. Amer. Math. Soc., 213 (1975), 373-390.

[24] J. Kozik, T. Krawczyk, P. Micek and W. T. Trotter, personal communication.

[25] T. Mészáros, P. Micek and W. T. Trotter, Boolean Dimension, Components and Blocks, https://arxiv.org/abs/1801.00288.

[26] P. Micek and B. Walczak, personal communication.

[27] K. Milliken, A Ramsey theorem for trees, J. Combin. Theory Ser. A, 26 (1979), 215-237.

[28] J.Nešetřil and P. Pudlák, A Note on Boolean dimension of posets, Irregularities of Partitions, Algorithms Combin. 8 (1989), 137-140.

[29] J. Spencer, Minimal scrambling sets of simple orders, Acta. Math. Hungar., 22 (1972), 349-353.

[30] N. Streib and W. T. Trotter, Dimension and height for posets with planar cover graphs, European J. Combinatorics, 35 (2014), 474-489.

[31] W. T. Trotter, Irreducible posets with arbitrarily large height exist, J. Combin. Theory Ser. A, 17 (1974), 337-344.

[32] W. T. Trotter, Inequalities in dimension theory for posets, Proc. Amer. Math. Soc., 47 (1975), 311-316.

[33] W. T. Trotter, Combinatorics and Partially Ordered Sets: Dimension Theory, The Johns Hopkins University Press, Baltimore, MD, 1992.

[34] W. T. Trotter and J. I. Moore, The dimension of planar posets, J. Combin. Theory Ser. B, 21 (1977), 51-67. 
[35] W. T. Trotter and B. Walczak, Boolean dimension and local dimension, Elec. Notes in Discrete Math., 61 (2017), 1047-1053.

[36] W. T. Trotter and R. Wang, Planar posets, dimension, breadth and the number of minimal elements, Order, 33 (2016), 333-346.

[37] T. Ueckerdt, personal communication.

[38] W. T. Trotter, B. Walczak and R. Wang, Dimension and cut vertices: An application of Ramsey theory, Connections in Discrete Mathematics, S. Butler, et al., eds., Cambridge University Press (2018) 187-199.

(Barrera-Cruz) Sunnyvale, CA

E-mail address: fidel.barrera@gmail.com

(Prag, Trotter) School of Mathematics, Georgia Institute of Technology, Atlanta, Georgia 30332

E-mail address: \{tprag3,trotter\}@math.gatech.edu

(Smith) Department of Mathematics and Computer Science, Davidson College, DavidSON, NC 28035

E-mail address: hcsmith@davidson.edu

(Taylor) Department of Mathematics, Stanford University, Stanford, CA 94305

E-mail address: libbytaylor@stanford.edu 